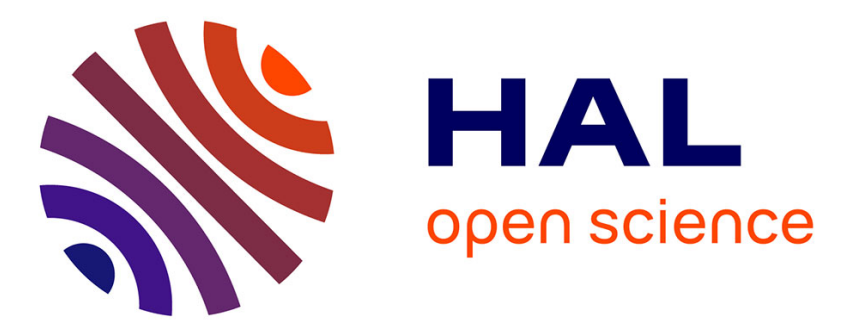

\title{
Enhanced runout and erosion by overland flow at low pressure and sub-freezing conditions: Experiments and application to Mars
}

Susan Conway, Michael P. Lamb, Matthew Balme, Martin C Towner, John Murray

\section{To cite this version:}

Susan Conway, Michael P. Lamb, Matthew Balme, Martin C Towner, John Murray. Enhanced runout and erosion by overland flow at low pressure and sub-freezing conditions: Experiments and application to Mars. Icarus, 2011, 211 (1), pp.443-457. 10.1016/j.icarus.2010.08.026 insu-02276892

\section{HAL Id: insu-02276892 \\ https://hal-insu.archives-ouvertes.fr/insu-02276892}

Submitted on 3 Sep 2019

HAL is a multi-disciplinary open access archive for the deposit and dissemination of scientific research documents, whether they are published or not. The documents may come from teaching and research institutions in France or abroad, or from public or private research centers.
L'archive ouverte pluridisciplinaire HAL, est destinée au dépôt et à la diffusion de documents scientifiques de niveau recherche, publiés ou non, émanant des établissements d'enseignement et de recherche français ou étrangers, des laboratoires publics ou privés. 


\section{Enhanced runout and erosion by overland flow at low pressure and subfreezing conditions: experiments and application to Mars}

3

\section{*Susan J. Conway}

Work done at: Earth and Environmental Sciences, Open University, Walton Hall, Milton Keynes MK7 6AA UK

Now at: Laboratoire de planétologie et géodynamique, CNRS UMR 6112, Université de Nantes, 2 rue de la Houssinière, BP 92208, 44322 Nantes cedex, France tel : +33 (0)2 51125570

Susan.Conway@univ-nantes.fr

\author{
Michael P. Lamb \\ MC 170-25 California Institute of Technology Pasadena, CA 91125 USA tel: +1 6263953612 \\ mpl@gps.caltech.edu
}

\section{Matthew R. Balme}

Earth and Environmental Sciences, Open University, Walton Hall, Milton Keynes MK7 6AA UK tel:+44 (0)1908 659776 fax:+44 (0)1908 655151 m.r.balme@open.ac.uk

\section{Martin C. Towner}

Impacts and Astromaterials Research Centre, Department of Earth Science and Engineering, Imperial College London, SW7 2AZ, UK. tel:+44 (0)20759 47326 fax:+44 (0) 2075947444

m.towner@imperial.ac.uk

\section{John B. Murray}

Earth and Environmental Sciences, Open University, Walton Hall, Milton Keynes MK7 6AA UK tel:+44 (0)1908659776 fax:+44 (0)1908655151 j.b.murray@open.ac.uk

*Corresponding author.

Running Head: Experimental Study of Erosion and Runout on Mars

Number of pages: 55

Number of Figures: 14 (+ 6 videos)

Number of Tables: 3 
We present the results of laboratory experiments to study the sediment transport and erosional capacity of water at current martian temperature and pressure. We have performed laboratory simulation experiments in which a stream of water flowed over test beds at low temperature $\left(\sim-20{ }^{\circ} \mathrm{C}\right)$ and low pressure $(\sim 7$ mbar $)$. The slope angle was $14^{\circ}$ and three sediment types were tested. We compared the erosive ability, runout and resulting morphologies to experiments performed at ambient terrestrial temperature $\left(\sim 20^{\circ} \mathrm{C}\right)$ and pressure ( 1000 mbar), and also to experiments performed under low pressure only. We observed that, as expected, water is unstable in the liquid phase at low temperature and low pressure, with boiling and freezing in competition. Despite this, our results show that water at low temperature and low pressure has an equivalent and sometimes greater erosion rate than at terrestrial temperature and pressure. Water flows faster over the sediment body under low temperature and low pressure conditions because the formation of ice below the liquidsediment contact inhibits infiltration. Flow speed and therefore runout distance are increased. Experiments at low pressure but Earth-ambient temperature suggest that flow speeds are faster under these conditions than under Earth-ambient pressure and temperature. We hypothesise that this is due to gas bubbles, created by the boiling of the water under low atmospheric pressure, impeding liquid infiltration. We have found that both basal freezing and low pressure increase the flow propagation speed - effects not included in current models of fluvial activity on Mars. Any future modelling of water flows on Mars should consider this extra mobility and incorporate the large reduction in fluid loss through infiltration into the substrate. 


\section{Motivation}

56

57

58

59

60

61

62

63

Many previous geomorphological studies have invoked liquid water as the agent for creating surface features on Mars. The current climate on Mars has both a temperature and pressure which are too low for stable water to exist, and similar climatic conditions have been assumed to have persisted for the majority of the Hesperian and Amazonian epochs (e.g., Marchant and Head, 2007). Outflow channels on Mars span a range of ages, from the Noachian into the Amazonian (e.g., Kieffer, 1992), and other examples of large-scale features that have been linked to the action of liquid water during this period include deltas (e.g., Kraal et al., 2008) and alluvial fans (e.g., Williams and Malin, 2008). Extremely recent, but smaller-scale surface features that have been attributed to the action of liquid water include kilometre-scale martian gullies (e.g., Malin and Edgett, 2000) and slope streaks (e.g., Kreslavsky and Head, 2009). The formation of all these features depends on the transport, erosion and deposition by liquid water, whose stability also depends on the temperature and pressure conditions on the surface. To understand the discharges, volume of water and timescales required to form these features requires an understanding of the effect of the ambient temperature and pressure conditions on the behaviour of water flowing over the martian surface. For calculating discharges, volumes and timescales of water flows, the effect of the metastability of liquid water is generally included in a general "fluid loss parameter", however this is usually poorly constrained. For example, when considering delta formation Kraal et al. (2008) use an upper limit of $50 \%$ discharge loss rate, which includes the effects of both infiltration and evaporation, but not freezing. In the modelling of recent gullies Heldmann et al. (2005), Pelletier et al. (2008) and Kolb et al. (2010) include a combined fluid loss parameter (which implicitly includes losses due to freezing, evaporation and infiltration). 
78 However, the fluid loss parameter adopted in these studies range from $10^{3}-10^{6} \mathrm{~mm} / \mathrm{h}$. Pelletier et al. note that the models of gully formation are particularly sensitive to this parameter, hence the estimated volumes and discharges of water required to form these features are too. Other potential effects of overestimating the fluid loss parameter include underestimates of erosion power and runout distance.

There have been several recent numerical and experimental studies that have investigated the sublimation and freezing of stationary bodies of water and brines under martian conditions, (e.g. Bryson et al., 2008; Chevrier and Altheide, 2008) but, although these experiments give important constraints on the behaviour of water under low pressure and temperature, their results cannot easily be extrapolated to flowing water. Only a few studies have specifically tried to investigate sediment transport under present-day martian conditions, for example, Védie et al. (2008) performed experiments designed to simulate the formation of Russell Crater's dune gullies under ambient Earth pressure and low temperature. No experiments to date have attempted to produce water flows under the low temperature and low pressure experienced on the present day martian surface.

Despite the obvious, yet poorly constrained effect of fluid loss due to freezing and evaporation of water under low temperature and pressure, other potential effects have previously only been briefly considered. For example Bargery et al (2005) and Leask et al. (2007) consider in theoretical terms the action of ice formation within the flow, which potentially acts to reduce the flow speed and erosion and to increase deposition. However, other unanswered questions include the possible effects of the formation of bubbles and/or ice at the base during the flow. Do these change the fluid dynamics and hence the erosion, transport, deposition, and runout distance of water and sediment under current martian conditions? To better constrain future modelling and to understand the potential factors influencing the erosion, sediment transport and runout of water flowing under martian 
conditions, a deeper understanding of the interaction between sediment and water under low temperature and pressure conditions is needed.

Herein we present a set of exploratory experiments to begin to fill this knowledge gap. In particular we explore the effect of Mars-like temperatures and pressures on overland flow of water over an erodible bed. First, we present methods of the low pressure and low temperature experimental setup, instrumentation and methods. Second, results from the experiments are presented which highlight the effects of freezing and boiling on flow runout, fluid loss, and erosion. Third, we present some simplified scaling analyses which explain parts of the results. Forth, implications for Mars surface processes are discussed.

\section{Method}

The experiments presented herein are exploratory in nature because they are the first set of experiments, to our knowledge, to investigate overland flow and erosion under combined Mars-like surface temperatures and pressures. The experiments are not meant to be exact replications of the martian surface. Instead, the goal is to isolate certain parameters that are probably different on Mars, as compared to Earth, and investigate their effect on fluid flow and sediment transport. In this contribution, we have chosen to investigate the effect of subfreezing substrate temperatures, fluid temperatures, atmospheric pressure, and sediment size. There are other variables that deserve experimental attention, such as changes in fluid properties due to solute concentrations (e.g., high density, viscous brines, Burt and Knauth, 2003), sediment mineralogy, and martian gravity. Exploring these other variables is beyond the scope of this contribution, however, because designing an experimental facility in which all possible variables can be explored is difficult and at times counter-productive. 


\subsection{Chamber description}

126 The sediment test bed was contained within a cylindrical low pressure chamber $2 \mathrm{~m}$ in length 127 and $\sim 1 \mathrm{~m}$ in diameter. The test bed was a $1 \mathrm{~m}$ long, $0.1 \mathrm{~m}$ deep rectangular metal tray of 128 trapezoidal cross section measuring $0.50 \mathrm{~m}$ across the base and $0.54 \mathrm{~m}$ across the top. A $\sim 5$ cm deep layer of various combinations of unconsolidated material was placed in this tray to form the sediment substrate. The tray was placed on a copper cooling plate and the whole test bed set at an angle of $14^{\circ}$ to the horizontal (Fig. 1). Water was introduced at the upper edge of the test bed and allowed to flow down and across the sediment substrate. All the experiments used water containing no dissolved salts. For the control experiments performed at ambient pressure, the water was introduced through a $14 \mathrm{~mm}$ diameter hose connected to a container $\sim 5 \mathrm{~m}$ above the chamber. For experiments at low pressure, the water was introduced from a calibrated container placed outside the chamber, at the same level as the source hose - the difference in pressure was enough to drive the water into the chamber. The flow rate was kept constant at 0.08 litres per second for all experiments. Thus each experiment lasted approximately 30 seconds and a total of 2.51 of water was used each time. Inside the chamber, the source hose was positioned centrally on the rim of the tray. Water was thus introduced onto the top of the sediment body; with a drop of approximately $3 \mathrm{~cm}$. Water was not introduced underneath or directly onto the surface of the sediment to avoid ice blockages forming in the hose. A solenoid valve within the end of the hose allowed external control over the release of water. A diffuser was located below the solenoid valve to dampen the horizontal velocity component of the water. There was no outlet for water at the end of the tray, just a backstop. The sediment substrate was chilled using a cooling plate in contact with the entire base of the tray. The cooling plate, a copper slab, was cooled by interior flow of liquid nitrogen. Baffles within the cold plate distributed the cooling effect of the liquid 
nitrogen throughout its area. The pressure in the chamber was actively controlled using a vacuum pump and was maintained at $\sim 7$ mbar for the low pressure experiments.

<FIGURE 1 HERE $>$

152

153

\subsection{Instrumentation}

Three pairs of thermocouples were placed within the sediment at $2 \mathrm{~cm}$ depth and $14 \mathrm{~cm}$ from the edges of the tray at the longitudinal distances marked on Fig. 1. Their output was recorded at one second intervals by a data logger. In all low temperature experiments the average temperature of the sediment bed was below $-20^{\circ} \mathrm{C}$ before the experiment was run, representing an above average, but not unexpected local surface temperature for Mars (e.g. Haberle et al., 2001). For six of the experiments the water temperature was pre-chilled to $5{ }^{\circ} \mathrm{C}$ and for three further experiments the water was pre-chilled to $0.5^{\circ} \mathrm{C}$.

All experimental runs were monitored and recorded using an internal and external webcam (with different view angles) and a digital camera. This allowed playback and detailed observations to be made of the evolution of the flow, the morphology, and the relative timings of events. The flow speed was estimated by noting the time taken for the flow to reach the end of the tray from the video recordings, with a measurement error of \pm 1 s. Once each experiment had finished, photographs were taken of the sediment surface. Exploratory excavations were made to investigate the sub-surface changes to the sediment body and to measure the thickness of frozen sediment, if present. For low temperature/low pressure experiments the chamber was opened only after the temperature on all thermocouples was observed to be dropping - this was taken as an indication that freezing of the water was complete - thus allowing the preservation of any sedimentary structures present.

Cross sections were measured with a surface profiler before and after the experiment to enable measurement of the volume of sediment transported. The profiles were measured at 
marked $10 \mathrm{~cm}$ intervals along the tray, including both ends. The profiler allowed the surface

of the test bed to be measured by a grid of $8 \times 11$ points, accurate to about $0.1 \mathrm{~cm}$ in height, before and after each run. After the experimental run was complete, further measurements at higher spatial resolution were made where the surface height changed abruptly - for example at the edges and tops of levees, channel walls, or at the ends of lobes. We measured both the channel width and the wetted width for each cross section. The wetted widths were not measured in the area where the flow ponded, hence we excluded all measurements within $20 \mathrm{~cm}$ of the backstop from the statistics and results. We measured channel depth from the cross profiles to estimate a flow depth to be used in the calculations of the Reynolds's $(\mathrm{Re})$ and Froude (Fr) Numbers. For these calculations we took the kinematic viscosity of water as $1.52 \times 10^{-6} \mathrm{~m}^{2} / \mathrm{s}$ and Earth's gravity as $9.8 \mathrm{~m} / \mathrm{s}^{2}$. The planimetric area for each flow was determined using a combination of orthorectified photographs and the data from the cross sections. Volumes of erosion and deposition were derived from these data as described in Section 4.

\subsection{Sediment characterisation}

We used two different sands to evaluate the effect of grain size and a poorly sorted material rock crush - to investigate the effects of a broad grain size distribution. Specifically, the substrates used were: (i) Leighton Buzzard DA 16/30, a medium sand, (ii) Leighton Buzzard RH T, a fine sand and, (iii) poorly sorted rock crush containing particles ranging in size from fine silt to gravel. The sands are both composed of quartz grains and their size distributions were measured by dry sieving (Atkinson, 2008). The rock crush is a mixture of crushed igneous rocks, including basalt and granite. The grain size distribution of the rock crush was measured using the wet sieve method and hydrometer to British Standard BS1377 Part 4:1990 by Soil Property Testing Ltd, Huntingdon, UK. Quantitative grain size data are shown in Table 1 and Fig. 2. 
<FIGURE 2 AND TABLE 1 HERE $>$

The permeability of each material was measured using the falling head method (Head, 1982) by Soil Property Testing Ltd, Huntingdon, UK and is shown in Table 1. We use the permeability of the materials as a minimum estimate of their infiltration rate. Under equilibrium conditions the infiltration rate approaches the permeability (Youngs, 1964), however the instantaneous infiltration rate of each of the materials is a function of both the permeability and the sorptivity of the material. For the two sands, it is reasonable to assume that a constant factor should apply, however for the rock crush, this factor could be slightly larger (Culligan et al., 2005). Bulk density, particle density and porosity (Table 1) were ascertained prior to permeability testing using the standard methods as described in Head (1982). The angle of repose of the materials was measured by gently forming a loose conical pile of sediment and averaging two measurements of the incline of the slopes formed. The angle of repose was very similar for the two sands $\left(33-35^{\circ}\right)$, but much greater for the rock crush $\left(41^{\circ}\right)$. The angularity of the sediments was determined by microscopy: the sand grains were sub-rounded to well-rounded in shape; the rock crush had sub-angular to angular grains. Grain compositions and grain size distributions have been shown to be widely variable on Mars from in-situ observations from Viking (Clark et al., 1977; Moore and Jakosky, 1989) through to the Mars Exploration Rovers (e.g. Cabrol et al., 2007; Jerolmack et al., 2006; Sullivan et al., 2008) and from remote sensing observations that use thermal inertia as a proxy for grain size (e.g. Fergason et al., 2006). Grain sizes range from clay-size (Pike et al., 2009) to boulders and can be very well sorted through to very poorly sorted. The materials used as simulants are somewhat more restricted (e.g. Peters et al., 2008; Sizemore and Mellon, 2008), but still have a range of physical and chemical properties. Although we are exploring the effects of material parameters, rather than simulating martian regolith per $s e$, the physical properties of the materials used in this study are certainly within the bounds 
of possible martian surface materials. Very fine material was avoided due to technical and health and safety restrictions, rather than its inapplicability to Mars.

\section{Results}

\subsection{Summary}

Table 2 provides a summary of the results for all the experiments performed in this study. For each sediment type, three experiments were performed at low temperature and low pressure, one was performed at room temperature but low pressure, and one performed at ambient pressure and room temperature. Within the low temperature/low pressure experiments, two were performed with water at $\sim 5^{\circ} \mathrm{C}$ and one with water at $\sim 0.5^{\circ} \mathrm{C}$. An example of the appearance of the sediments at the end of each experiment is shown in Fig. 3, with labels to explain the terms used in the text.

Our experiments had a range of Reynolds Numbers (Table 2): only the medium and fine sands were fully turbulent $(\operatorname{Re}>1000)$ for their maximum values of $\operatorname{Re}$. The flows in the sands were usually partially turbulent and the flows over the rock crush were always laminar $(\operatorname{Re}<100)$. The Froude Numbers of our flows (Table 2) ranged from $0.06-1.19$, but only flows in the rock crush experienced critical $(\mathrm{Fr}>1)$ conditions. The rest of the flows were subcritical $(\mathrm{Fr}<1)$ and the fine sand had the lowest range of Froude Number $(0.14-0.69)$.

$<$ FIGURE 3 and TABLE 2 HERE $>$

\subsection{Observations: low temperature and low pressure experiments}

\subsubsection{All Sediment Types}

For all sediment types the water was seen to exude gas bubbles (e.g. video 1) and to form ice on introduction into the chamber, indicating simultaneous boiling and freezing. Observations of the sediment body after the experiments were completed confirm that water was able to 
infiltrate only a small depth into the bed before it froze, forming an icy-sediment lens over which the rest of the flow progressed (Fig. 3). The sediments underneath were still dry. Boiling resulted in the formation of bubbles within the ice and the frozen sediments. Where water collected at the end of the tray (e.g. video 2), the resulting ice was extremely bubblerich and opaque on top with an underlying translucent, bubble-free layer. This structure is similar to those described by Cheng and Lin (2007) and Bargery (2008) in experiments performed with standing bodies of water at low temperature and low pressure.

$<$ VIDEO 1 and VIDEO 2 HERE $>$

\subsubsection{Fine and Medium Sand}

256

For the sand substrates the flow initially spread out laterally across the surface at the top of the tray and then progressed down the slope along one or more principal paths (video 1 and video 3). Bubbling water was seen to flow over the surface and, towards the end of the experiment, formed distinct channels (Figs. 3 and 4A). In the case of the fine sand the flow continuous and did not migrate laterally (compare video 1 fine sand and video 3 medium sand), depositing low lateral levees. In both cases, the channels and levees were linear rather than sinuous. When the flow encountered the backstop, water and sediment spread laterally and backed up, collecting into a pool extending 10-20 cm upstream from the bottom of the tray (Fig. 4A). This ponded water bubbled vigorously in most cases, forming large bubbles ( $\sim 1 \mathrm{~cm}$ for medium sand and $1-5 \mathrm{~cm}$ for medium sand), until the surface froze (video 2).

\section{<FIGURE 4 and VIDEO 3 HERE $>$}

The fine sand formed more small lateral lobes than the medium sand (Figs. 4B and 4C). For the $\sim 0.5^{\circ} \mathrm{C}$ water runs the deposits were rougher and formed a fan of icy slush. In these experiments almost no water ponded at the end. Runs that used the warmer $5{ }^{\circ} \mathrm{C}$ water 
often showed ponding of water at the end of the test bed that resulted in a wedge of ice. The icy wedges at the end of the flow had dry sediment underneath, showing that the flow had not penetrated to the base of the tray.

\subsubsection{Rock Crush}

The flow initially spread out laterally as for the sands. However, the flow then progressed as multiple digitate lobes (Fig. 4D), which then quickly coalesced into a sheet flow, rather than channelized flow (in contrast to flow over the sand beds) as shown in video 4 and Fig. 5. On one occasion, small but detectable channels and fans did form, but these were within the sheet-like flow. It is notable that the depositional fan in this case was entirely composed of the finer material; coarser material was not transported. As the flow encountered the backstop the water backed up to $20-25 \mathrm{~cm}$ upstream and ponded. This water bubbled gently with small bubbles forming (1-5 mm) until an ice sheet formed over the top (video 5).

\section{<FIGURE 5, VIDEO 4 and VIDEO 5 HERE>}

Close observation revealed that the flow front progressed by travelling around the larger clasts, before inundating them as the flow matured. In cross section, the icy sediment lens contained a concentration of coarser clasts at the top (Fig. 4E), indicating the surface had been washed free of fines. Bubbles were not observed breaking the surface in the rock crush, but were observed in the ice lens and ice wedge deposits at the end of the tray. Water was observed to pond at the end of the tray irrespective of the initial water temperature. The ice wedge which then formed at the end of the tray penetrated through the sediment to the base of the tray, except when the water was cooled to $\sim 0.5^{\circ} \mathrm{C}$, when $1-2 \mathrm{~cm}$ of dry sediment was left underneath. 


\subsection{Observations: control experiments performed at 1) Earth ambient}

\subsubsection{All Sediment Types}

297 The water was able to infiltrate into the sediments for all the experiments. There were therefore some obvious differences from the experiments performed at low temperature and pressure:

(i) flows were slower to progress down slope for a given sediment type (Table 2).

(ii) there was no ponding of water at the end of the tray.

(iii) wet haloes of sediment formed around the flows, extending downwards as

\subsubsection{Fine and Medium Sand}

Compared to the low temperature/low pressure experiments the flows in sands remained confined laterally, both initially and throughout the flow duration, Fig. 5. For the fine sand the flows had some lateral migration, but much less than the low temperature/low pressure runs (video 1). The lateral migration of flows across the medium sand was even more limited (video 3). Both flows built lateral levees and were somewhat pulsing in nature. In cases where the flow encountered the backstop a fan of sediment built up, propagating laterally ( $15 \mathrm{~cm}$ ) and upstream. None of the flows on the medium sand substrate reached the end of the tray under ambient temperature and pressure conditions. In all cases water infiltrated downwards to the base of the tray beneath all the flows. During the ambient temperature/low 
pressure experiment both sands contained bubbles and had surface blisters. The bubbles and blisters were present in the percolation halo as well as along the flow path.

321 The flow for the rock crush was very similar in style to the experiments at low temperature/low pressure (video 4). Initially the flow spread both downstream and laterally, and continued to do so as the flow progressed. The flow propagated in all directions forming a radial flow front, elongate in the downstream direction. In contrast to the low temperature/low pressure experiments, the flow was not initially digitate. The wet sediment surface was observed to bubble during the ambient temperature/low pressure experiments (video 6). For the ambient temperature and pressure experiment the flow did not reach the end of the test bed (video 4) and it barely reached the backstop in the low pressure experiment. Not enough flow reached the backstop for it to have significant influence on the progress of the flow (video 6).

<VIDEO 6 HERE $>$

The flows on the rock crush substrate progressed much more slowly for both the ambient temperature experiments than they did for the cold runs (Table 2). The flow was more channelized in the uppermost portion than for the low temperature/low pressure experiments. Infiltration in both cases resulted in the water penetrating to the base of the tray under the flow lobe. Despite the boiling observed in motion during the ambient temperature/low pressure experiments, no bubbles were preserved within the rock crush because there was no ice to preserve them. 


\section{Data analysis}

340

341

342

343

344

345

346

347

\subsection{Volume calculation}

The $\mathrm{x}, \mathrm{y}$ and $\mathrm{z}$ coordinates from the measured cross profiles were interpolated into a gridded surface using the Kriging method in Surfer 8 software. This method has provision to allow for anisotropy in data collection (a greater density of sampling was used post-experiment in some cases). A $1 \mathrm{~cm}$ grid size was chosen as appropriate for the wavelength of changes observed and applied to all the surfaces. To calculate the volume of erosion and deposition for each experiment the pre-experiment surface was subtracted from the post-experiment surface. The results for the overall volumes are given in Table 2 and an example of the spatial results is mapped in Fig. 7.

\section{$<$ FIGURE 7 HERE >}

The deposition volumes are much larger than the erosion volumes for low temperature/low pressure experiments. Most of the additional volume can be accounted for by the ponded water at the base of the flow, and by large cavities that formed in the ice wedge as a result of boiling. Within the bounds of error ( $\pm 1 \mathrm{~mm}$ in height measurements) the erosion and deposition balance out for the ambient temperature/ambient pressure experiments (Fig. 8C). The data show consistent excess in deposition volume for all the ambient temperature/low pressure experiments: this may represent an increase in volume through incorporated gas, although we note that these values are comparable to the estimated measurement error.

Because the deposition data include additional ice, water and gas, we used the erosion volume to estimate the volume of sediment transported. This erosion volume was derived for each experiment simply by summing all the pixels in each difference map that had negative displacements. From this we generated an erosion rate, based on the estimated volume of 
material removed normalised to the tray area and the duration of the flow. Using the estimated removed sediment volume, the material porosity and the volume of water we made an estimate of the sediment concentration in the flows. From the spatial distribution of erosion and deposition we also determined the "erosion distance" - the horizontal distance that each flow travelled before changing from net erosion to net deposition (Fig. 7). The erosion distance is another measure of the erosional ability of the flow. This was performed by dividing the tray area into segments (Fig. 7) and summing all the erosion and depositional pixels within each segment. This determined whether each segment was dominated by erosion or by deposition, as well as the net erosion, or deposition. The horizontal distance at which the transition occurred was determined graphically. In reality this is a minimum estimate, because some of the surface lowering by erosion is countered by ice expansion and bubble formation, which masks some of the areas which actually had small net erosion.

\subsection{Erosion}

377 For all the experiments this erosion rate was between 0.002 and $0.055 \mathrm{~mm} . \mathrm{s}^{-1}$ and the erosion 378 distance was between 50 and $650 \mathrm{~mm}$ from the source of the flow. In general, the rock crush shows much lower erosion (rate, or distance) than either of the sands (Fig. 9 and Table 2). This result is consistent with the results of Shields (1936) and Kirchner et al. (1990) that larger particles and more angular particles require more stress to move. For the low temperature/low pressure experiments, all sediment types had higher erosion rate and distance when using the warmer water (Fig. 9). However, the patterns of erosion rates for the different experiments varied between each sediment type: (1) in the sub-freezing experiments the erosion rate and distance in the medium sand was on average greater than in the ambient experiments, (2) the fine sand shows a similar trend, but less marked and, (3) for rock crush 
the erosion rate was lowest for the low temperature/low pressure with colder water, and all the other experiments have higher and very similar erosion rates.

\section{$<$ FIGURES 8 and 9 HERE $>$}

For the subfreezing experiments the erosional parts of the flow had a thinner ice lens

391 than the depositional parts of the flow (Figs. 7 and 8). The ice lens was thickest where deposition was greatest - usually at the end of the tray (Figs. 7 and 8). The icy-sediment lens formed at the base of the flow ranged from $0.5-3.5 \mathrm{~cm}$ thick for both the sand types and was thinner $(0.5-1.0 \mathrm{~cm})$ and more uniform for the rock crush.

\subsection{Runout distances}

The runout distances were calculated by projecting the flow speed (as calculated by the time for the flow front to reach the base of the tray) over the duration of the experiment. In all cases the runout distance is greater for each material type under sub-freezing conditions than under ambient temperature conditions (Fig. 10). This agrees with the qualitative observations of ponding occurring at the end of the subfreezing experiments, but not occurring at ambient

401

402

403

404

405 temperatures (documented in Sections 3.2 and 3.3). This effect is most marked in the medium sand. In addition when comparing the ambient temperature experiments performed at different pressures, the runout distances for the sands seem to be greater at low pressure than at ambient pressure (Fig. 10). However, this result should be treated with some caution as only a limited number of experiments were performed. 


\section{Discussion}

408

409

410

411

412

413

414

415

416

417

\subsection{Transport dynamics under low temperature and low pressure}

The formation of an ice lens at the base of the flow retarded infiltration, leading to more surface flow and therefore faster down slope flow propagation. We infer the lack of infiltration from the presence of dry sediments beneath the ice lens and from the pooling of excess water at the end of the tray. Infiltration experiments performed on soils under ambient terrestrial pressure conditions by McCauley et al. (2002) showed a similar distinct decrease in infiltration rate for freezing soils. If our test bed had been longer, the flows under freezing conditions would have had a significantly greater runout distance than those under ambient temperatures, as indicated by our calculations in Section 4.3. Freezing temperatures therefore have a fundamental affect on the behaviour of the flow, if not on the actual erosion rate.

We can estimate the depth to the freezing front by using Fourier's law of heat conduction. Assuming that the water is in contact with a semi-infinite plain of cold homogenous material (whereas in reality it has infiltrated into the pores of a granular mixture), under steady-state conditions, we can simplify Fourier's law to a heat loss per unit area $(q)$ which gives:

$$
q=\Delta E / \Delta t=k A\left(T_{1}-T_{2}\right) / x
$$

where $T_{1}\left(5^{\circ} \mathrm{C}\right)$ is the temperature of the water, $T_{2}\left(-20^{\circ} \mathrm{C}\right)$ the temperature of the substrate, $k$ the thermal conductivity of the water $(0.58 \mathrm{~W} / \mathrm{mK}), A$ the area of contact, $x$ the thickness of the material, $\Delta E$ the energy change and $\Delta t$ the time duration. If we assume that a thickness of one pore space must freeze to halt infiltration, we can use the pore space as the thickness, $x$. This is the distance over which the temperature must to be reduced to zero and hence we can calculate how much energy must be lost $(\Delta E)$. We need to account for the energy loss due to both the temperature drop and the enthalpy of fusion, for which we use a specific heat 
capacity, $C=4.210 \mathrm{~kJ} / \mathrm{kg} . \mathrm{K}$, a standard enthalpy of fusion, $H=333.55 \mathrm{~kJ} / \mathrm{kg}$ and a density, $\rho$

$432=1000 \mathrm{~kg} / \mathrm{m}^{3}$ for water at just above $0{ }^{\circ} \mathrm{C}$. Hence:

$$
\Delta E=\rho x\left(H+C T_{1}\right)
$$

434

435

436

437

438

439

440

441

442

443

444

445

446

447

448

449

450

451

452

453

454

and combining Eqs. (1) and (2) and rearranging gives:

$$
\Delta t=\rho x^{2}\left(H+C T_{1}\right) / k A\left(T_{1}-T_{2}\right)
$$

By using Eq. (3), we can estimate for each sediment type how much time is required to freeze such a layer and to what depth the water should have infiltrated when this occurs (using the permeability values listed in Table 1). The calculation is laid out for each material in Table 3. Despite the large number of simplifying assumptions, the depths to the bottom of the ice layer are broadly supported by our observed ice thicknesses (Fig. 11), both in terms of ranking and order of magnitude. However, our calculations over-estimate the depth of penetration into the medium sand and underestimate for the fine sand and rock crush. This could be due to an under-estimate of the instantaneous infiltration rates for the fine sand and rock crush (sorptivity is higher for smaller pores), an over-estimate of the pore size for the medium sand, or the violation of the other assumptions inherent in the calculation (planar continuous material and steady state conditions).

\section{$<$ TABLE 3 \& FIGURE 11 HERE $>$}

Starting from this mechanism we can build a simple process model. A schematic diagram representing the important stages in the evolution of the flow is shown in Fig. 12. The ice barrier forces the flow to be in the regime of saturation overland flow (Dunne and Leopold, 1978), because the depth to saturation is restricted by the ice lens. Under ambient conditions this regime is only experienced by the medium sand. In this case the depth to the base of the tray is so great that only limited amounts of overland flow occurs. If the medium sand was infinitely deep, the flow would not have propagated very far at all $(\sim 10 \mathrm{~cm})$. 
455 Hence, the greatest difference is seen for the runout and erosion for the medium sand. For the

456 fine sand and rock crush the discharge is sufficient to counteract the losses by infiltration, and

457 overland flow continues ("Horton overland flow": Horton, 1945). Hence the transition to

458 saturation overland flow under sub-freezing conditions increases the runout, but does not

459 always affect the erosion. The reason for the variations in erosion-rate dependence is 460 explored below.

\section{<FIGURE 12 HERE >}

It would be expected that the formation of a basal icy-lens at such shallow depths would retard erosion as it turns a cohesionless substrate into one with cohesion. However, the propagation of the freezing front is counterbalanced by the erosion rate of the unfrozen, yet saturated material (Fig. 12- $t_{4}$ ). It would be expected that once the saturated substrate above the growing ice has been removed, that thermal erosion would come into play. Thinner ice sheets were observed nearer the top of the tray (Figs. 7 and 8) and in the channels and this could be explained using thermal erosion arguments. In addition, we observed that the warmer, $5{ }^{\circ} \mathrm{C}$ water onto the cold substrate caused more erosion than the colder water at $\sim 0.5$ ${ }^{\circ} \mathrm{C}$ (Fig. 13). This can be explained using both arguments of faster propagation of the freezing front and less efficient thermal erosion. The rates of thermal erosion calculated from experimental and numerical modelling results of Costard et al. (1999) and Randriamazaoro et al. (2007), range upwards from $0.4 \mathrm{~mm} / \mathrm{min}\left(6.7 \times 10^{-6} \mathrm{~m} / \mathrm{s}\right)$. However, Randriamazaoro et al.

474 (2007) showed that thermal erosion increases with Re and the flow regimes in their study 475 have much greater Reynolds numbers $(\operatorname{Re}>6345)$ compared to our flows' Reynolds numbers 476 (5-1645; Table 2). Hence, their minimum rate provides an absolute maximum when applied 477 to our flows and, because it is much lower than our erosion rates $\left(1.56 \times 10^{-4}-\right.$ 478 $3.58 \times 10^{-3} \mathrm{~m} / \mathrm{min}$ ), we infer that the thermal erosion mechanism is not usually dominant. 
It is generally expected that erosion rates should be greater for greater flow rates, but

480

481

482

483

484

485

486

487

488

489

490

491

492

493

494

495

496

497

498

499

500

501

this was not always the case in our experiments. This could be due to the freezing bed

introducing two competing effects: it increases the flow rate, yet also impedes the erosion by freezing the particles to the bed (hence the slower thermal erosion mechanism becomes dominant). The dominance of one effect over the other is probably related to the freezing rate. As shown in Fig. 13 the erosion decreases as the difference in temperature between sediment and water decreases (the lower the difference the colder the water). Hence, the armouring of the bed is most effective at reducing erosion when the temperature difference is lower, with the water closer to freezing resulting in a faster propagation of the freezing front.

\section{<FIGURE 13 HERE>}

\subsection{Flow runout distances}

Another way of looking at the dynamics of these flows is to consider the mass balance of the flow. If we consider a discharge $(q)$ per unit width, $w$, then this results in the following:

$$
q=q_{i n}-V_{i} x-V_{e w} x-V_{f r} x
$$

where $q_{i n}$ is the initial discharge, $x$ is the distance from the outlet, $V_{i}$ is the rate of loss due to infiltration, $V_{e v}$ the rate of loss due to evaporation and $V_{f r}$ the rate of loss due to freezing. This assumes steady flow conditions with no lateral changes in width. These approximations are not valid for our experiments, however a full unsteady model would require a 3D morphodynamic model. This would include conservation of momentum, conservation of sediment, and constitutive equations for these highly concentrated $(20 \%$ for fine sand and 30 $\%$ for medium sand), self channelized flows. Such an attempt is beyond the scope of this analysis. Instead of a full solution, our goal is to provide a simple framework in which to assess the relative contributions of discharge, freezing, evaporation and infiltration to the 
flow runout distances. Despite these simplifying assumptions, the analysis yields insightful, albeit qualitative, results, as detailed below.

Using the mass balance Eq. (4), we can consider the length over which the flow discharge falls to zero, from this we can ascertain:

$$
\mathrm{L}=q_{i n} /\left(V_{i}+V_{e v}+V_{f r}\right)
$$

where $L$ is the total length of the flow.

From the experiments to investigate the evaporation of standing bodies of water under martian atmospheric pressure and at $0^{\circ} \mathrm{C}$ by Sears and Moore (2005) the value of $V_{e v}$ should be $\sim 2.0 \times 10^{-7} \mathrm{~m} / \mathrm{s}$. In our experiments, $V_{f r}$ changed as a function of downstream distance as shown by the increasing ice lens thickness in Figs. 4, 7 and 8. To estimate the range in $V_{f r}$, we use both the minimum and maximum value of thickness of sediment that froze at the base of the channels over $30 \mathrm{~s}$ for the sands and the rock crush. Hence, considering also the material porosity (but not the included bubbles), gives mean freezing rates ranging from $6.6 \times 10^{-4}$ to $1.6 \times 10^{-4} \mathrm{~m} / \mathrm{s}$ for fine sand, from $6.1 \times 10^{-4}$ to $2.3 \times 10^{-4} \mathrm{~m} / \mathrm{s}$ for medium sand and from $2.7 \times 10^{-4}$ to $6.0 \times 10^{-5} \mathrm{~m} / \mathrm{s}$ for rock crush. From Table 1 infiltration rates range from $5.21 \times 10^{-3}$ to $2.23 \times 10^{-4} \mathrm{~m} / \mathrm{s}$. Therefore, it can be seen that evaporation is relatively unimportant when compared to the other losses, by at least three orders of magnitude. When no freezing occurs during the flow $V_{f r}$ is zero and $V_{i}$ is at its maximum. Conversely under sub-freezing conditions $V_{i}$ approaches zero and the loss term is dominated by $V_{f r}$.

Using the above values for the freezing rates, evaporation rates and infiltration rates, we have used Eq. (5) to predict the expected runout distance for each of our experiments. In the subfreezing experiments we set $V_{i}$ equal to zero and in the experiments performed at room temperature, $V_{f r}$ was set to zero. Figure 14 is a plot of this predicted runout distance against the runout distance as calculated from the flow speed. There is good agreement between the two runout distances for the ambient temperature and pressure experiments. The runout 
distance is greater for the ambient temperature/low pressure experiments than predicted by

528 Eq. (5), a possible explanation for this is presented in Section 5.3. The predicted runout distance for the sub-freezing experiments is also generally an underestimate. The predicted runout using a minimum freezing rate (as indicated by the maximum vertical extent of the error bars in Fig. 14) produces a much better match to the calculated runout distances for the rock crush, but an overestimate for the two sands.

$<$ FIGURE 14 HERE $>$

\subsection{Influence of low pressure}

Our experimental data suggest that pressure is not as important as temperature for controlling the gross behaviour of the flows. However, the flow propagation speed was greater at low pressure than at ambient pressure. For example, the flows in the experiments performed with medium sand propagated to the end of the tray at ambient temperature/low pressure, but only propagated to $\sim 50 \mathrm{~cm}$ under ambient temperature/ambient pressure (Table 2). The effect is less marked (but still apparent) for the rock crush and the fine sand. A possible explanation

541 for enhanced flow at low pressure is that the formation of bubbles within the sediment 542 inhibits infiltration as the water boils. This effect was noted by Prunty and Bell (2007) who found unexpectedly low infiltration rates in their low-pressure infiltration experiments. It is well established that formation of bubbles from exsolved gases can greatly reduce aquifer permeability, for example Ronen et al. (1989) found a reduction of infiltration rates in sands from $45 \mathrm{~m}$ /day to $2 \mathrm{~m}$ /day resulting from biotic bubble formation and Amos and Ulrich Mayer (2006) found a reduction of up to $25 \%$ from abiotic bubble formation.

As detachment bubble size for boiling water increases with decreasing atmospheric pressure, it is possible that the bubble size of water on the martian surface is equivalent to or 
552 pressures of $<3$ mbar and found bubble detachment diameters of $0.3-0.5 \mathrm{~mm}$. Hence it is 553 certainly possible that bubble detachment size is greater than the pore size in our

554 experiments. Another possible explanation might be that small bubbles in the flow caused the 555 flow to be less dense, again reducing infiltration - although we note that this also should 556 cause flow propagation to be slower and is contrary to the observations.

\section{$557 \quad 5.4 \quad$ Implications at field scale}

558 Two features on Mars, gullies and slope streaks, could be forming at present and hence the results from our experiments could throw some light on their formation processes. Gullies on Mars are kilometre-scale features that resemble gullies that form on Earth due to overland flow of liquid water, or highly concentrated flows of sediment and water (debris flow). They have been widely studied as such since their discovery by Malin and Edgett (2000). Initially they were proposed to have formed by the outflow of water from a subsurface aquifer (Heldmann and Mellon, 2004; Malin and Edgett, 2000). However, recent observations on morphology, distribution and their setting within landform assemblages (e.g. Balme et al., 2006; Dickson and Head, 2009) has strongly suggested that they originate from melting of ice under recent climate excursions. Other explanations for their origin include dry (or carbon dioxide assisted) granular flow (e.g. Pelletier et al., 2008), but these mechanisms do not produce all of the key morphologies and do not explain their distribution. Key to both the aquifer model and the melting models is the efficiency of water in transporting sediment under martian surface temperature and pressure. having lower albedo, although some have higher albedo) that propagate downhill, being diverted around obstacles and affected by topography (e.g. Sullivan et al., 2001). They form (Aharonson et al., 2003) on Mars in time periods of less than 10 years. Slope streaks were 
577 first seen in Viking Orbiter images (e.g. Morris, 1982) and were found to be associated with 578 dusty areas on the planet (e.g. Ferguson and Lucchitta, 1984). These features have primarily 579 been interpreted as being formed by a dry mass wasting process (e.g. Chuang et al., 2007), 580 however some recent work has indicated that liquid water might be involved in their 581 formation (Kreslavsky and Head, 2009).

Although our experiments are not replicates of the martian surface, it is useful to use 583 Eq. (5), with the rates calculated from our experiments, to estimate the discharge that might be required to generate flows of this type over longer distances. Although mass balance (i.e., Eq. 5) should hold on Mars, there are caveats to directly applying the rates from our experiments to Mars, which are discussed in detail in Section 5.5. For sub-freezing conditions, similar to our experiments, when $V_{i} \sim 0$, gullies or slope streaks of $1 \mathrm{~km}$ in length and $20 \mathrm{~m}$ in width would require a discharge of between $670 \mathrm{ls}^{-1}$ and $130001 \mathrm{~s}^{-1}$, depending on the sediment type and freezing rate, for flow to occur from top to bottom. However, under non-freezing conditions, when $V_{f r}=0$, discharges in this system would need to be increased to between $4500 \mathrm{ls}^{-1}$ and $100000 \mathrm{ls}^{-1}$ depending on the sediment type. Some slope streaks have smaller dimensions and thus smaller discharge requirements, for example slope streaks of $5 \mathrm{~m}$ wide and $50 \mathrm{~m}$ long would require 8 to $1161 \mathrm{~s}^{-1}$ with a freezing substrate and 56 to $1300 \mathrm{ls}^{-1}$ without. The large ranges in discharges from our calculations emphasise the strong influence that infiltration rates have on the fluid loss parameter and on overall runout distances, both in terms of material type and in terms of the presence of an impermeable layer 597 (in our calculations an ice layer formed by the flow). Impermeable layers could also be 598 formed by permafrost or shallow bedrock and hence would not necessarily have a large freezing loss associated with them. However, these impermeable layers would be expected to 600 be found at greater depths, hence the reduction in infiltration would be less marked and occur with a time delay. 

et al. (2005) estimate $30000 \mathrm{ls}^{-1}$ for a generic gully, Hart et al. (2009) estimate $750-83000$ $1 \mathrm{~s}^{-1}$ for bankfull discharge from gully measurements at Lyot crater and Parsons and Nimmo (2010) give an estimate of $45000 \mathrm{ss}^{-1}$ from modelling sediment transport to generate a generic gully. Our mass-balance calculations based on our experimental results broadly support these flow rates. However, our calculations suggest that such large discharges are not required if the system is freezing. Heldmann et al. (2005) invoke these large flow rates to compensate for freezing and evaporative losses and to explain the formation of deep, wide channels in single events. Hart et al. (2009) generated large discharges to fulfil their assumption of bankfull discharge, without consideration of loss parameters. Parsons and Nimmo (2010) do not use a fluid loss parameter, as they consider losses as insignificant over the duration of gully formation. Our experiments emphasise the importance of considering infiltration rates, omitted from both these studies, when performing this kind of calculation. modelling gullies on Mars and has a great influence on the runout distance and morphology of the resulting flow (Kolb et al., 2010; Pelletier et al., 2008). Our work shows that under sub-freezing conditions there is some fluid loss through freezing, but also shows that fluid loss is reduced through the inhibition of infiltration. Our experiments highlight that evaporative losses are not important compared to losses due to freezing and infiltration and that careful consideration of these terms will be necessary in future modelling. The minimum

622 fluid loss values used by Kolb et al. (2010) and Pelletier et al. (2008) are at the maximum of our estimated fluid loss and only our highest infiltration rate (medium sand) approaches the

624 fluid loss parameter used by Heldmann et al. (2005), and even then solely under non-freezing 625 conditions. We maintain that further work is required to accurately define the quantitative 
626 limits of the fluid loss term under martian temperature and pressure for use in modelling 627 studies.

\subsection{Caveats to up-scaling the experimental results.}

629 Our experiments were designed to investigate the effect of Mars-like pressure and 630 temperature on overland flow and sediment transport. Although we feel the results are robust, care must be taken in extrapolating the results herein to natural systems that lie outside the parameter space investigated. This is in part because designing and conducting experiments within a low temperature and low temperature facility are necessarily at a scale smaller than most natural systems of interest and so explore a limited range of parameter space. Below we elaborate on these limitations and discuss future opportunities in experimentation.

Open-channel flows are typically scaled dynamically using the Reynolds number and the Froude number (e.g., Chow, 1959). Each of our experiments had different ranges of Reynolds numbers (Re) and within each experiment Re spanned a range of values (Table 2).

Only one experiment in the medium and one in the fine sand were fully turbulent $(\operatorname{Re}>1000)$ where Re was at its maximum. The flows in the sands were usually partially turbulent and the flows over the rock crush were always laminar $(\operatorname{Re}<100)$. Larger flows with fully turbulent Re might be expected to have different runout lengths, not only because of their greater size, but also due to changes in bed friction (Chow, 1959) and the rate of turbulent energy dissipation to heat (Tennekes and Lumley, 1972). This notwithstanding, we saw no major trend in our results with Re, indicating that perhaps these are second order effects as compared to the changes in infiltration rate caused by freezing. Moreover, it has been argued that many similarities exist in sediment bed morphodynmaics between laminar flows and turbulent flows (Lajeunesse et al., 2010). space from subcritical to critical conditions. Subcritical Froude number are much more 
common in sediment-transport systems on Earth (Grant, 1997), but supercritical flows can

652

653

654

655

656

657

658

659

660

661

662

663

664

665

666

667

668

669

670

671

672

673

674

occur, especially on steep slopes. For martian gullies, models have explored a range of Froude numbers including supercritical conditions (Heldmann et al., 2005; Kolb et al., 2010; Parsons and Nimmo, 2010; Pelletier et al., 2008). Since the transition to supercritical flow can significantly change flow hydraulics (e.g., by allowing hydraulic jumps) future work is needed to explore this area of parameter space in more detail.

The above discussion of Re and Fr implicitly assumes dilute $(c<<1)$ Newtonian-flow conditions. It is possible that some of the gullies on Mars are carved by highly concentrated non-Newtonian flows (i.e., debris flows, e.g., Lanza et al., 2010) or dry avalanches (Treiman, 2003). In some of our experiments the sediment concentrations were high (Table 2), and showed some non-Newtonian behaviour including granular snouts of flows and levees on the sides of channels. It is less clear how to up-scale runout lengths and erosion of such flows, given the complex interplay between particle-particle interactions and pore pressure (Hsu et al., 2008; Iverson, 1997). These experiments were not designed to simulate gully formation by dry avalanching.

The effect of changes in gravity on overland flow and sediment transport has been explored by others (Burr et al., 2006; Komar, 1980). In general, it has been found that flows will move slower under lower gravity, but that sediment weighs less, so that sediment transport rates on Earth and Mars scale similarly. It is also expected that infiltration rates will be slower under reduced gravity (Chan et al., 2004). However, we suspect that all of these effects that result from different gravity for Mars conditions (by roughly a factor of three from Earth conditions) should be small (by several orders of magnitude) in comparison to the effect of reduced infiltration due to freezing, and therefore should not change substantially the experimental findings. 

property that exerts the greatest influence on the experiments is the permeability of the material. A secondary effect was the ability of the flow to entrain certain sizes of particles. Where the flow was not able to entrain particles in our experiments (rock crush), flow spreading occurred. This counter-acted the effect of reduced infiltration rate which would otherwise have acted to further increase the runout distance. If we were to use a sediment that was highly impermeable, with small to average grain size, a situation could occur in which runout is maximised. However, we predict in this case that erosion would be slow, because the bed would freeze quickly and erosion would progress mainly by slower thermal erosion as the bed is melted again.

We also only tested one inclination angle of the test bed. Higher inclination should 686 increase the flow speed and slightly reduce the infiltration effects (Dingman, 1994). The temperature of the test bed and fluids should also influence the results. Given a lower sediment temperature the freezing front should occur at a shallower depth. We did vary the input water temperature $\left(5^{\circ} \mathrm{C}\right.$ or $\left.0.5^{\circ} \mathrm{C}\right)$, which also decreases the temperature difference. However, the effects of the more rapid formation of an ice lens were possibly masked by the effects of ice particles forming within the flow.

Using different fluids could have a significant effect on fluid behaviour. Various compounds have been proposed to form solutions on Mars, which could facilitate the stability of water at low temperatures. Suggested compounds include: perchlorates (Catling et al., 2009; Hecht et al., 2009), Calcium Chloride (Knauth and Burt, 2002), Sodium Chloride 696 (Sears et al., 2002), and Ferric Sulphate (Chevrier and Altheide, 2008) brines; organics (Jean et al., 2008) and acids (Benison et al., 2008). These all have a higher viscosity than pure water. For example Chevrier et al. (2009) found the viscosity of ferric sulphate solutions to be between $7.0 \times 10^{-3}$ and 4.6 $\mathrm{Pa}$ s at temperatures of $285-260 \mathrm{~K}$. An increase in viscosity 
700

701

702

703

704

705

706

707

708

709

710

711

712

713

714

715

716

717

718

719

720

721

722

would act to slow the flow rate for flows with $\operatorname{Re}<10^{3}$ and decrease infiltration (e.g., Jarsjö et al., 1997; Lin et al., 2003). Flow rates for fully turbulent flows $\left(\operatorname{Re}>10^{3}\right)$ are independent of fluid viscosity(Tennekes and Lumley, 1972). The stability of these solutions at low temperatures would prevent the formation of a basal ice lens and might even promote thermal erosion of any permafrost at the base of the flow (Andersland et al., 1996). These factors combined suggest that very careful investigation of the inherent infiltration rate of the sediment type is needed in these cases. If infiltration is very large and there is no formation of a basal ice lens, then discharges would have to be very high for fluids to be able to flow overland and form features such as gullies. This could make the above fluids implausible when considering gullies on isolated topography.

\section{Conclusions}

These experiments highlight some potential pitfalls when considering water flows on the surface of Mars. Specifically, when using a fluid loss parameter in modelling, careful consideration should be given to the factors influencing infiltration in the flow bed, i.e. (1) its temperature (2) the infiltration rate of the unconsolidated material, (3) the presence or absence of an impermeable layer and (4) the depth of such a layer if present.

We have found that water flowing over a freezing substrate behaves very differently from water flowing over a warm bed. In the former case, water freezes at a shallow depth in the substrate, impeding infiltration, causing the flow to propagate faster and further that it would under ambient terrestrial conditions. This suggests that fluvial flow features on Mars could be formed by volumes of liquid an order of magnitude less than for similar-length flows on the Earth. 

conditions also act to change the flow dynamics, but less dramatically than low temperature.

We found that flows are faster with a greater runout at low pressure/ambient temperature. We hypothesise that infiltration is impeded by the formation of bubbles at the base of the flow, but further work needs to be done to confirm this mechanism. Again, this suggests that smaller volumes of water are required to create long flows on Mars than on the Earth.

Our experiments indicate that previous estimates of gully discharges are within the upper estimates of those indicated by our analysis, but that the assumptions inherent in these earlier calculations have underestimated the factors influencing the fluid loss parameter, especially the infiltration rate. Further experimental work needs to be done to further constrain the factors influencing fluid loss on the surface of Mars.

\section{Acknowledgements}

We thank two anonymous reviewers for their comments which greatly improved this manuscript. This work would not have been possible without a postgraduate studentship grant from the U.K. Natural Environment Research Council (NERC). We gratefully acknowledge the support of the staff at the Open University's Research Design and Engineering Facility and technical staff at Planetary and Space Science Research Institute. We thank Luther Beegle for sharing his raw data on grainsize analysis of JSC-1 and MMS Mars simulants and Karl Atkinson for allowing us to use his grainsize analyses results. We thank the staff at Soil Property Testing Ltd., Huntingdon, UK for performing the permeability testing and grainsize analysis. We are grateful to Helen Townsend for her assistance in the laboratory. 
Aharonson, O., Schorghofer, N., Gerstell, M. F., 2003. Slope streak formation and dust deposition rates on Mars. J. Geophys. Res. Planets. 108, 5138.

Amos, R. T., Ulrich Mayer, K., 2006. Investigating the role of gas bubble formation and entrapment in contaminated aquifers: Reactive transport modelling. J. Contam. Hydrol. 87 (1-2), 123-154.

Andersland, O. B., Wiggert, D. C., Davies, S. H., 1996. Frozen soil subsurface barriers: Formation and ice erosion. J. Contam. Hydrol. 23 (1-2), 133-147.

Balme, M., Mangold, N., Baratoux, D., Costard, F., Gosselin, M., Masson, P., Pinet, P., Neukum, G., 2006. Orientation and distribution of recent gullies in the southern hemisphere of mars: Observations from High Resolution Stereo Camera/Mars Express (HRSC/MEX) and Mars Orbiter Camera/Mars Global Surveyor (MOC/MGS) data. J. Geophys. Res. Planets. 111 (E5), doi:10.1029/2005JE002607

Bargery, A. S., Wilson, L., Mitchell, K. L., 2005. Modelling Catastrophic Floods on the Surface of Mars. Lunar Planet. Sci. Conf. 36, no. 1961.

Benison, K. C., LaClair, D., Walker, J., 2008. Physical sedimentology experiments with sulfuric acid solutions: Implications for Mars? Earth. Planet. Sci. Lett. 270 (3-4), 330-337.

Bryson, K. L., Chevrier, V., Sears, D. W. G., Ulrich, R., 2008. Stability of ice on Mars and the water vapor diurnal cycle: Experimental study of the sublimation of ice through a fine-grained basaltic regolith. Icarus. 196 (2), 446-458.

Burr, D. M., Emery, J. P., Lorenz, R. D., Collins, G. C., Carling, P. A., 2006. Sediment transport by liquid surficial flow: Application to Titan. Icarus. 181 (1), 235-242.

Burt, D. M., Knauth, L. P., 2003. Electrically conducting, Ca-rich brines, rather than water, expected in the Martian subsurface. J. Geophys. Res. Planets. 108, 8026.

Cabrol, N. A., Grin, E. A., Herkenhoff, K., Richter, L., Science, T. A., 2007. Soil Sedimentology, Textures and Dynamics at Gusev Crater from Spirit's Microscopic Imager. Lunar Planet. Sci. Conf. 38, no. 1784.

Catling, D. C., Claire, M. W., Quinn, R. C., Zahnle, K. J., Clark, B. C., Kounaves, S., Hecht, M. H., 2009. Possible Atmospheric Origins of Perchlorate on Mars. Lunar Planet. Sci. Conf. 40, no. 1567.

Chan, T.-Y., Hsu, C.-S., Lin, S.-T., 2004. Factors Affecting the Significance of Gravity on the Infiltration of a Liquid into a Porous Solid. J. Porous Mater. 11 (4), 273-277.

Chevrier, V., Altheide, T. S., 2008. Low temperature aqueous ferric sulfate solutions on the surface of Mars. Geophys. Res. Lett. 35 (L22101), L22101

Chevrier, V. F., Ulrich, R., Altheide, T. S., 2009. Viscosity of Liquid Ferric Sulfate Solutions and Application to the Formation of Gullies on Mars. Lunar Planet. Sci. Conf. 40, no. 1424.

Chow, V. T., 1959. Open-channel hydraulics. McGraw-Hill College, New York.

Chuang, F. C., Beyer, R. A., McEwen, A. S., Thomson, B. J., 2007. HiRISE observations of slope streaks on Mars. Geophys. Res. Lett. 34 (20), doi:10.1029/2007GL031111.

Clark, B. C., III, et al., 1977. The Viking X ray fluorescence experiment - Analytical methods and early results. J. Geophys. Res. 82, 4577-4594.

Costard, F., Aguirre-Puente, J., Greeley, R., Makhloufi, N., 1999. Martian fluvial-thermal erosion: Laboratory simulation. J. Geophys. Res. Planets. 104 (E6), 14091-14098.

Culligan, P. J., Ivanov, V., Germaine, J. T., 2005. Sorptivity and liquid infiltration into dry soil. Adv. Water Res. 28 (10), 1010-1020.

Dickson, J. L., Head, J. W., 2009. The formation and evolution of youthful gullies on Mars: Gullies as the late-stage phase of Mars' most recent ice age. Icarus. 204 (1), 63-86.

Dingman, S. L., 1994. Physical Hydrology. Prentice-Hall, Upper Saddle River, N. J.

Dunne, T., Leopold, L. B., 1978. Water in Environmental Planning. W. H. Freeman, San Francisco.

Fergason, R. L., Christensen, P. R., Kieffer, H. H., 2006. High-resolution thermal inertia derived from the Thermal Emission Imaging System (THEMIS): Thermal model and applications. J. Geophys. Res. Planets. 111, E12004

Ferguson, H. M., Lucchitta, B. K., 1984. Dark streaks on talus slopes, Mars. Planetary Geology and Geophysics Program Report, 188-190. 
Grant, G. E., 1997. Critical Flow Constrains Flow Hydraulics in Mobile-Bed Streams: A New Hypothesis. Water Resour. Res. 33 (2), 349-358.

Haberle, R. M., McKay, C. P., Schaeffer, J., Cabrol, N. A., Grin, E. A., Zent, A. P., Quinn, R., 2001. On the possibility of liquid water on present-day Mars. J. Geophys. Res. Planets. 106 (E10), 23317-23326.

Hart, S. D., Gulick, V. C., Parsons, R. A., Barnhart, C. J., 2009. Gully Slopes and Discharges on Lyot Crater's Central Peak. Lunar Planet. Sci. Conf. 40, no. 2349.

Head, K. H., 1982 Soil classification and compaction tests. In: K. H. Head, (Ed.), Manual of Soil Laboratory Testing Vol. 1. Pentech Press, London.

Hecht, M. H., et al., 2009. Perchlorate in Martian Soil: Evidence and Implications. Lunar Planet. Sci. Conf. 40 , no. 2420.

Heldmann, J. L., Mellon, M. T., 2004. Observations of martian gullies and constraints on potential formation mechanisms. Icarus. 168 (2), 285-304.

Heldmann, J. L., Toon, O. B., Pollard, W. H., Mellon, M. T., Pitlick, J., McKay, C. P., Andersen, D. T., 2005. Formation of Martian gullies by the action of liquid water flowing under current Martian environmental conditions. J. Geophys. Res. Planets. 110 (E5), doi:10.1029/2004JE002261.

Horton, R. E., 1945. Erosional development of streams and their drainage basins; hydrophysical approach to quantitative morphology. Geol. Soc. Am. Bull. 56 (3), 275-370.

Hsu, L., Dietrich, W. E., Sklar, L. S., 2008. Experimental study of bedrock erosion by granular flows. J. Geophys. Res. Earth Surf. 113 (F2), doi:10.1029/2007JF000778.

Iverson, R. M., 1997. The physics of debris flows. Rev. Geophys. 35 (3), 245-296.

Jarsjö, J., Destouni, G., Yaron, B., 1997. On the relation between viscosity and hydraulic conductivity for volatile organic liquid mixtures in soils. J. Contam. Hydrol. 25 (1-2), 113-127.

Jean, J. S., Yang, C. H., Lee, M. J., Lee, M. K., Chien, M. H., 2008. Potential antifreeze compounds in present-day Martian seepage groundwater. Terr. Atmos. Ocean. Sci. 19 (3), 279-289.

Jerolmack, D. J., Mohrig, D., Grotzinger, J. P., Fike, D. A., Watters, W. A., 2006. Spatial grain size sorting in eolian ripples and estimation of wind conditions on planetary surfaces: Application to Meridiani Planum, Mars. J. Geophys. Res. Planets. 111 (12), doi:10.1029/2005JE002544.

Kieffer, H. H., 1992. Mars. University of Arizona Press, Tucson.

Kirchner, J. W., Dietrich, W. E., Iseya, F., Ikeda, H., 1990. The variability of critical shear stress, friction angle, and grain protrusion in water-worked sediments. Sedimentology. 37 (4), 647672.

Knauth, L. P., Burt, D. M., 2002. Eutectic brines on Mars: Origin and possible relation to young seepage features. Icarus. 158 (1), 267-271.

Kolb, K. J., Pelletier, J. D., McEwen, A. S., 2010. Modeling the formation of bright slope deposits associated with gullies in Hale Crater, Mars: Implications for recent liquid water. Icarus. 205 (1), 113-137.

Komar, P. D., 1980. Modes of sediment transport in channelized water flows with ramifications to the erosion of the Martian outflow channels. Icarus. 42, 317-329.

Kraal, E. R., van Dijk, M., Postma, G., Kleinhans, M. G., 2008. Martian stepped-delta formation by rapid water release. Nature. 451 (7181), 973-976.

Kreslavsky, M. A., Head, J. W., 2009. Slope streaks on Mars: A new "wet" mechanism. Icarus. 201, 517-527.

Lajeunesse, E., et al., 2010. Fluvial and submarine morphodynamics of laminar and near-laminar flows: a synthesis. Sedimentology. 57 (1), 1-26.

Lanza, N. L., Meyer, G. A., Okubo, C. H., Newsom, H. E., Wiens, R. C., 2010. Evidence for debris flow gully formation initiated by shallow subsurface water on Mars. Icarus. 205 (1), 103-112.

Leask, H. J., Wilson, L., Mitchell, K. L., 2007. Formation of Mangala Valles outflow channel, Mars: Morphological development and water discharge and duration estimates. J. Geophys. Res. Planets. 112, doi: 10.1029/2006JE002851.

Lin, C., Greenwald, D., Banin, A., 2003. Temperature dependence of infiltration rate during large scale water recharge into soils. Soil Sci Soc Am J. 67 (2), 487-493.

Malin, M. C., Edgett, K. S., 2000. Evidence for recent groundwater seepage and surface runoff on Mars. Science. 288 (5475), 2330-2335. 
Marchant, D. R., Head, J. W., 2007. Antarctic dry valleys: Microclimate zonation, variable geomorphic processes, and implications for assessing climate change on Mars. Icarus. 192, 187-222.

McCauley, C. A., White, D. M., Lilly, M. R., Nyman, D. M., 2002. A comparison of hydraulic conductivities, permeabilities and infiltration rates in frozen and unfrozen soils. Cold Reg. Sci. Technol. 34 (2), 117-125.

Moore, H. J., Jakosky, B. M., 1989. Viking landing sites, remote-sensing observations, and physical properties of Martian surface materials. Icarus. 81, 164-184.

Morris, E. C., 1982. Aureole deposits of the Martian volcano Olympus Mons. J. Geophys. Res. 87, 1164-1178.

Parsons, R. A., Nimmo, F., 2010. Numerical modeling of Martian gully sediment transport: Testing the fluvial hypothesis. J. Geophys. Res. 115 (E6), doi:10.1029/2009JE003517.

Pelletier, J. D., Kolb, K. J., McEwen, A. S., Kirk, R. L., 2008. Recent bright gully deposits on Mars: Wet or dry flow? Geology. 36 (3), 211-214.

Peters, G. H., Abbey, W., Bearman, G. H., Mungas, G. S., Smith, J. A., Anderson, R. C., Douglas, S., Beegle, L. W., 2008. Mojave Mars simulant--Characterization of a new geologic Mars analog. Icarus. 197 (2), 470-479.

Pike, W. T., Sykulska, H., Vijendran, S., Phoenix Microscopy, T., 2009. Fractal Analysis of the Microstructure of the Martian Soil at the Phoenix Landing Site. Lunar Planet. Sci. Conf. 40, no. 1909.

Prodanovic, V., Fraser, D., Salcudean, M., 2002. Bubble behavior in subcooled flow boiling of water at low pressures and low flow rates. Int. J. Multiphase Flow. 28 (1), 1-19.

Prunty, L., Bell, J., 2007. Infiltration Rate vs. Gas Composition and Pressure in Soil Columns. Soil Sci. Soc. Am. J. 71 (5), 1473-1475.

Randriamazaoro, R., Dupeyrat, L., Costard, F., Gailhardis, E. C., 2007. Fluvial thermal erosion: Heat balance integral method. Earth Surf. Process. Landforms. 32 (12), 1828-1840.

Ronen, D., Berkowitz, B., Magaritz, M., 1989. The development and influence of gas bubbles in phreatic aquifers under natural flow conditions. Transp. Porous Media. 4 (3), 295-306.

Sears, D. W. G., Benoit, P. H., McKeever, S. W. S., Banerjee, D., Kral, T., Stites, W., Roe, L., Jansma, P., Mattioli, G., 2002. Investigation of biological, chemical and physical processes on and in planetary surfaces by laboratory simulation. Planet. Space Sci. 50 (9), 821-828.

Sears, D. W. G., Moore, S. R., 2005. On laboratory simulation and the evaporation rate of water on Mars. Geophys. Res. Lett. 32 (16), 4.

Shields, I. A., 1936. Application of Similarity Principles and Turbulence Research to Bed-

Load Movement. California Institute of Technology, Pasadena, CA.

Sizemore, H. G., Mellon, M. T., 2008. Laboratory characterization of the structural properties controlling dynamical gas transport in Mars-analog soils. Icarus. 197 (2), 606-620.

Sullivan, R., Thomas, P., Veverka, J., Malin, M., Edgett, K. S., 2001. Mass movement slope streaks imaged by the Mars Orbiter Camera. J. Geophys. Res. Planets. 106 (E10), 23607-23633.

Sullivan, R., et al., 2008. Wind-driven particle mobility on Mars: Insights from Mars Exploration Rover observations at "El Dorado" and surroundings at Gusev Crater. J. Geophys. Res. Planets. 113 (E6), doi:10.1029/2008JE003101.

Tennekes, H., Lumley, J. L., 1972. A first course in turbulence. The MIT Press, Cambridge, MA and London, England.

Treiman, A. H., 2003. Geologic settings of Martian gullies: Implications for their origins. J. Geophys. Res. Planets. 108 (E4), doi:10.1029/2002JE001900.

Védie, E., Costard, F., Font, M., Lagarde, J. L., 2008. Laboratory simulations of Martian gullies on sand dunes. Geophys. Res. Lett. 35 (L21501), doi:10.1029/2008GL035638.

Williams, R. M. E., Malin, M. C., 2008. Sub-kilometer fans in Mojave Crater, Mars. Icarus. 198, 365383.

Youngs, E. G., 1964. An Infiltration Method of Measuring the Hydraulic Conductivity of Unsaturated Porous Materials. Soil Sci. 97 (5), 307-311. 
908 Figure 1. Diagram of the experimental apparatus. Dark bounding box represents the hypobaric

909 chamber. For the experiments under low pressure, the pressure was lowered and maintained using two 910 vacuum pumps. The tray containing the substrate was tilted at an angle of $14^{\circ}$ and the water was

911 introduced from an external reservoir and its release onto the sediment controlled via a solenoid valve.

912 Six thermocouples (TC) were placed within the sediment body to monitor the temperature of the

913 sediment at a depth of $2 \mathrm{~cm}, 14 \mathrm{~cm}$ from the tray edge. The sediment was cooled using liquid nitrogen

914 circulated thorough a hollow copper plate located beneath the aluminium tray containing the

915 substrate. An internal webcam was used to monitor the progression of the experiment. 
917 Figure 2. Plot showing the grain size distribution of the sediments used in this study. The results of

918 Atkinson (2008) are given for the fine and medium sands and the results from Soil Property Testing 919 Ltd., Huntingdon, UK for the rock crush.

920 
921 Figure 3. Photograph of experiment under low temperature and pressure in fine sand (low P\&T,

922 No.3), illustrating terms used in text. The source is located at the top of the image and the flow ran

923 from top to bottom. Dotted line indicates the extent of the tray (lower end is not included in the

924 photograph because of the angle of capture). Solid black lines indicate the position of the cross

925 sections and the arrows connect the corresponding locations on both photographs. The scale bars on

926 the smaller photographs are $2 \mathrm{~cm}$ in length.

927 
Figure 4. A. Photograph of low pressure and low temperature experiment in medium sand after completion (low P\&T, No.1). Photograph taken facing source of the flow, with the width of the tray being $54 \mathrm{~cm}$. Water has ponded at the end of the tray (bottom of the picture) forming a bubble-rich ice wedge. Note also that the flow starts to form a fan towards the end of the tray and this continues beneath the ice (not shown). B. Photograph of low pressure and low temperature experiment in fine sand after completion (low P\&T, No.2). Photograph taken facing source of the flow, maximum width of lobe $\sim 10 \mathrm{~cm}$. The flow has formed multiple small lateral lobes and the rough appearance of the surface is caused by bubbles. C. Photograph of low pressure and low temperature experiment in medium sand after completion (low P\&T, No.2). Photograph taken facing source of the flow, maximum width of lobe $\sim 17 \mathrm{~cm}$. The flow has formed one large lateral lobe and the lighter colour of the flow is due to ice that condensed onto the flow when the chamber was opened to the atmosphere. D. Photograph of low pressure and low temperature experiment in rock crush, about $5 \mathrm{~s}$ into the experiment (low P\&T, No.1). Photograph taken facing source of the flow, with the width of the tray being $54 \mathrm{~cm}$. Notice the digitate margins to the flow, which stabilised into sheet flow later in the experiment. E. Photograph of cross section through icy-lens of rock crush experiment under low pressure and low temperature (low P\&T, No.2). Scale bar in top-right corner is $5 \mathrm{~mm}$. Notice that the largest grains are concentrated at the top of the section. F. Photograph of cross section through a flow in fine sand at ambient pressure and ambient temperature (amb P\&T, No.1). $f$ indicates the flow width and $w$ indicates the wetted width - a substantial wetted halo has formed due to lateral water migration. The photo was taken facing the base of the tray, with the section located $\sim 40 \mathrm{~cm}$ from the end of the tray. 
950 Figure 5. Flow wetted width normalised to the flow width at ambient temperature and pressure against

951 median sediment grain size for all experiments. Vertical error bars are based on a $5 \%$ uncertainty in

952 calculating the flow area from the cross sections and orthophotos. Horizontal error bars are $10 \%$

953 uncertainty associated with measuring the median grain size. 
955 Figure 6. Flow planimetric area normalised to the flow area at ambient temperature and pressure 956 against median sediment grain size for all experiments. Vertical error bars are based on a $10 \%$

957 uncertainty in calculating the flow width from the cross sections. Horizontal error bars are $10 \%$ 958 uncertainty associated with measuring the median grain size. 
960 Figure 7. A: isopach map generated from measured cross profiles, with solid lines delineating the

961 segments used to calculate the net deposition values displayed in B. B: associated plot of net

962 deposition volume (negative values are erosion) with dotted lines connecting the segments in A to the

963 associated data point in B. In addition the measured frozen sediment thicknesses are recorded on the

964 same plot and the black arrow indicates the position used to estimate the erosion distance (i.e. where

965 the deposition volume goes from negative to positive). This plot is for the low pressure and

966 temperature experiment in fine sand (low P\&T-3), and the same experiment is shown in Fig. 3. 
968 Figure 8. A \& B: Plots of distance from source against net deposition (negative deposition indicates 969 erosion) and ice-sediment thickness for low temperature and pressure experiment over medium sand 970 (A, low P\&T, No.2) and rock crush (B, low P\&T, No.3). C: plot of distance from source against net 971 deposition and cumulative deposition for an ambient temperature and pressure experiment in fine sand 972 (amb P\&T, No.1). Grey vertical lines in all plots mark the zero line of deposition. 
974 Figure 9. A: Erosion rate normalised to the erosion rate at ambient temperature and pressure against

975 median sediment grain size for all experiments. Vertical error bars are based on a $1 \mathrm{~mm}$ vertical

976 uncertainty in measuring the change of height of the sediment surface, which leads to an error in the

977 volume calculation and hence erosion rate. B: Erosion distance normalised to the erosion distance at

978 ambient temperature and pressure against median sediment grain size for all experiments. Vertical

979 error bars are based on a $50 \mathrm{~mm}$ uncertainty in measuring the erosion distance. Horizontal error bars

980 are $10 \%$ uncertainty associated with measuring the median grain size. 
982 Figure 10. Estimated runout distance from flow speeds normalised to the estimated runout at ambient

983 temperature and pressure against sediment grain size for all experiments. Vertical error bars are

984 calculated uncertainties based on a $1 \mathrm{~s}$ error in measuring the time taken for the flow to reach the end

985 of the tray, which provides an error for the speed and hence runout distance. Horizontal error bars are

$98610 \%$ uncertainty associated with measuring the median grain size. 
988 Figure 11. Predicted ice thickness against measured ice thickness, for three selected experiments at 989 low pressure and temperature (medium sand low P\&T-2, fine sand low P\&T-3, rock crush low P\&T990 3). Horizontal error bars represent the maximum and minimum ice thickness measured. Vertical error 991 bars are set at $50 \%$, because the $50 \%$ uncertainty in the pore-size dominates over the uncertainty of 992 the infiltration rate and temperature difference. Diagonal grey line is the 1:1 line. 
994 Figure 12. Sketch to illustrate progression of flow at low pressure and low temperature. $t_{0}$ dry

995 sediment column. $t_{1}$ water is introduced and infiltration dominates over downstream flow. $t_{2}$ sufficient

996 ice forms to inhibit infiltration and downstream flow starts to dominate. $t_{3}$ and $t_{4}$ the freezing front

997 propagates upwards into the saturated sediment above and the flow removes saturated sediment from

998 the top. $t_{5}$ the flow stops and the remaining saturated sediment (if any) freezes, leaving a frozen

999 channel bed. 
1001 Figure 13. A: Erosion rate against temperature difference between sediment and water for 1002 experiments at low pressure and temperature. B: Erosion distance against temperature difference 1003 between sediment and water for experiments at low pressure and temperature. 
1004 Figure 14. Runout distance predicted from freezing rate calculations against runout distance

1005 calculated from observed flow speeds, during all experiments. The calculated runout distances shown 1006 for the low pressure and temperature experiments are averages of the three experiments. 
1008 Video 1: Comparison between experiment at low pressure and temperature (left - low P\&T, No.3)

1009 and ambient conditions (right - amb P\&T, No.1) for fine sand. View is from the base of the tray

1010 towards the source, filmed from the outside of the chamber. 
1012 Video 2: Side-view (from the left-hand-side looking from base-to-source, from outside the chamber) 1013 of experiment low P\&T, No.1 with medium sand under low pressure and low temperature conditions. 1014 The video has been cut into a time series to illustrate the evolution of the ponded water. Notice that 1015 over time the wetted sediment surface gets lighter in colour over time and the frost formed on the 1016 outside of the tray retreats with time (lower right of view). 
1018 Video 3: Comparison between experiment at low pressure and temperature (left - low P\&T, No.3)

1019 and ambient conditions (right - Amb P\&T, No.2) for medium sand. View is from the base of the tray 1020 towards the source, filmed from the outside of the chamber. 
1022 Video 4: Comparison between experiment at low pressure and temperature (left - low P\&T, No.1) 1023 and ambient conditions (right - Amb P\&T, No.1) for rock crush. View is from the base of the tray 1024 towards the source, filmed from the outside of the chamber. 
1026 Video 5: Side-view (from the left-hand-side looking from base-to-source, from outside the chamber)

1027 of experiment low P\&T, No.3 with rock crush under low pressure and low temperature conditions.

1028 The video has been cut into a time series to illustrate the evolution of the ponded water. 
1030 Video 6: Side-view (from the left-hand-side looking from base-to-source, from outside the chamber) 1031 of experiment Low P, No.1 with rock crush under low pressure and ambient temperature conditions. 1032 The puffs of sediment are possibly a result of trapped air being released from under the sediment 1033 surface. Notice that the surface of the flow continues to bubble after the flow has stopped. 
Figure 1

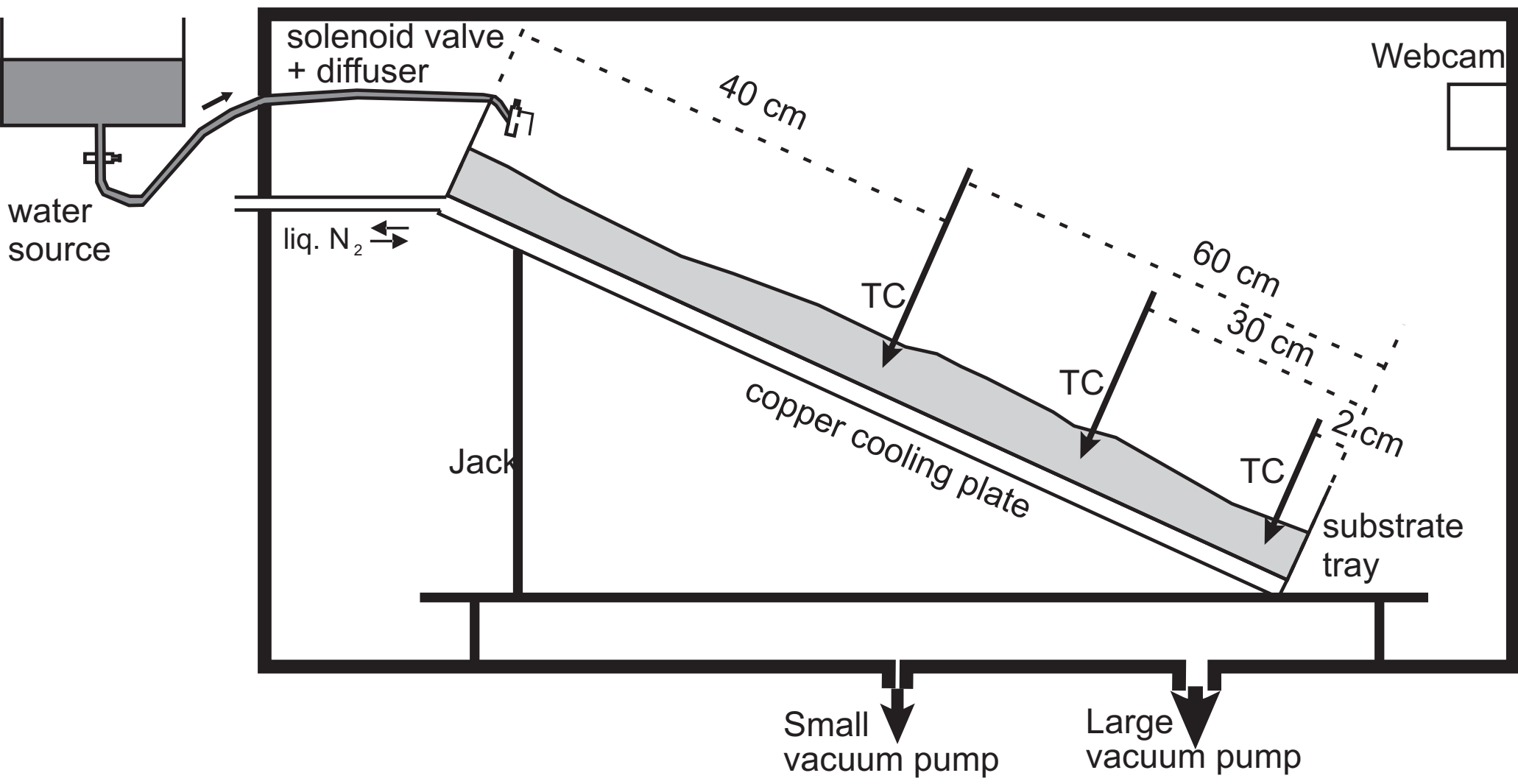




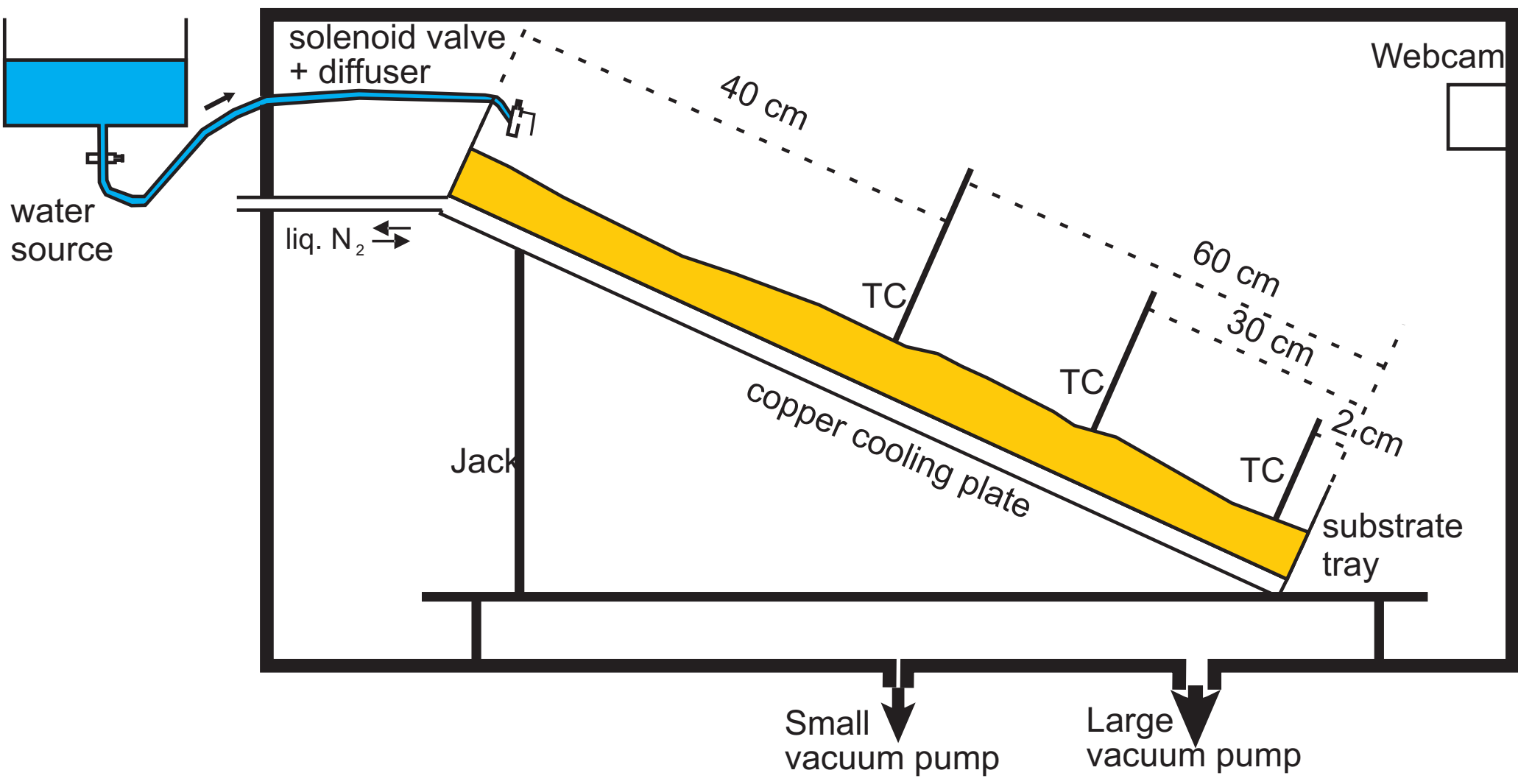




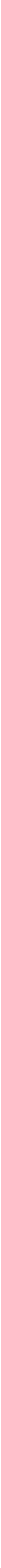




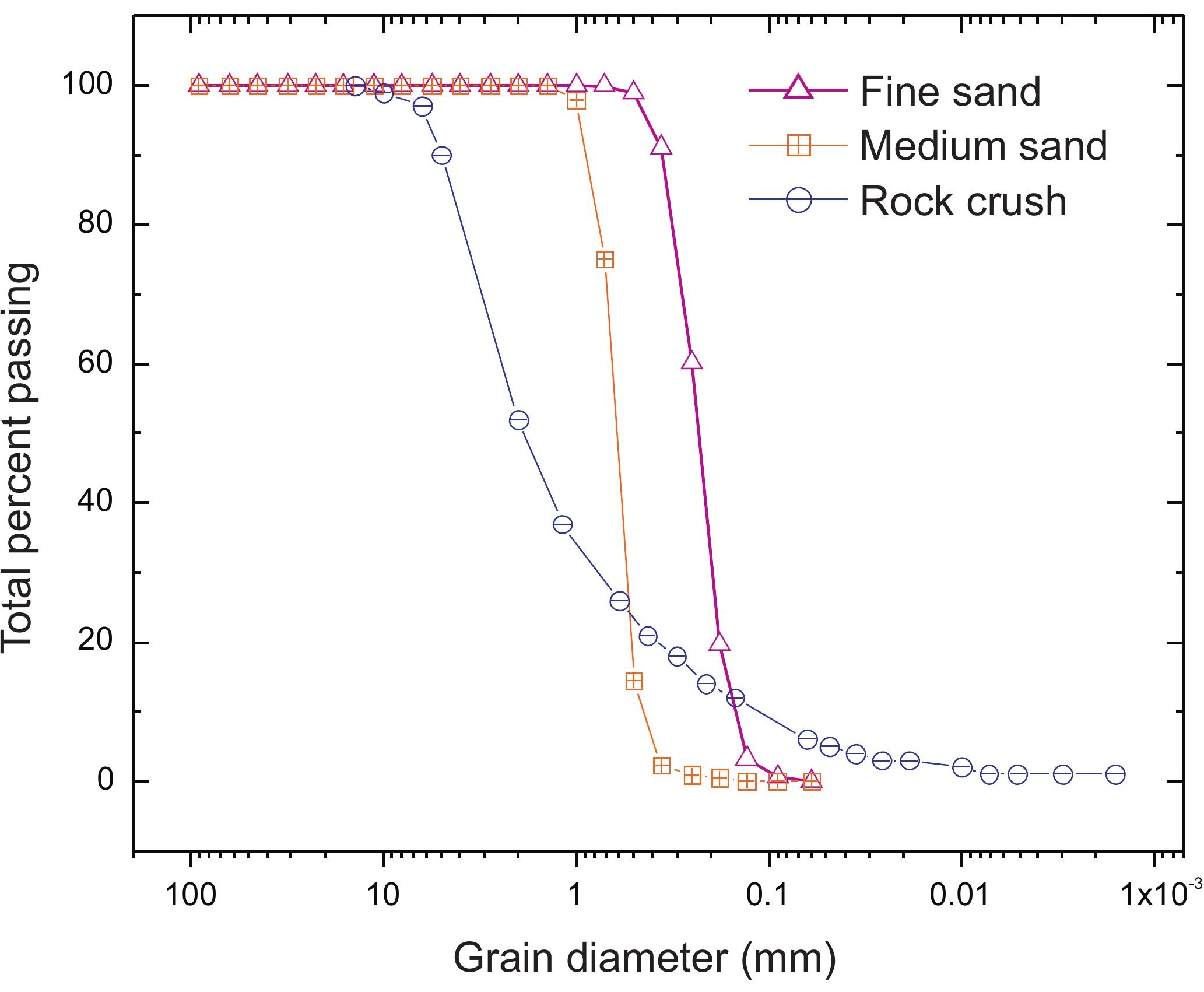




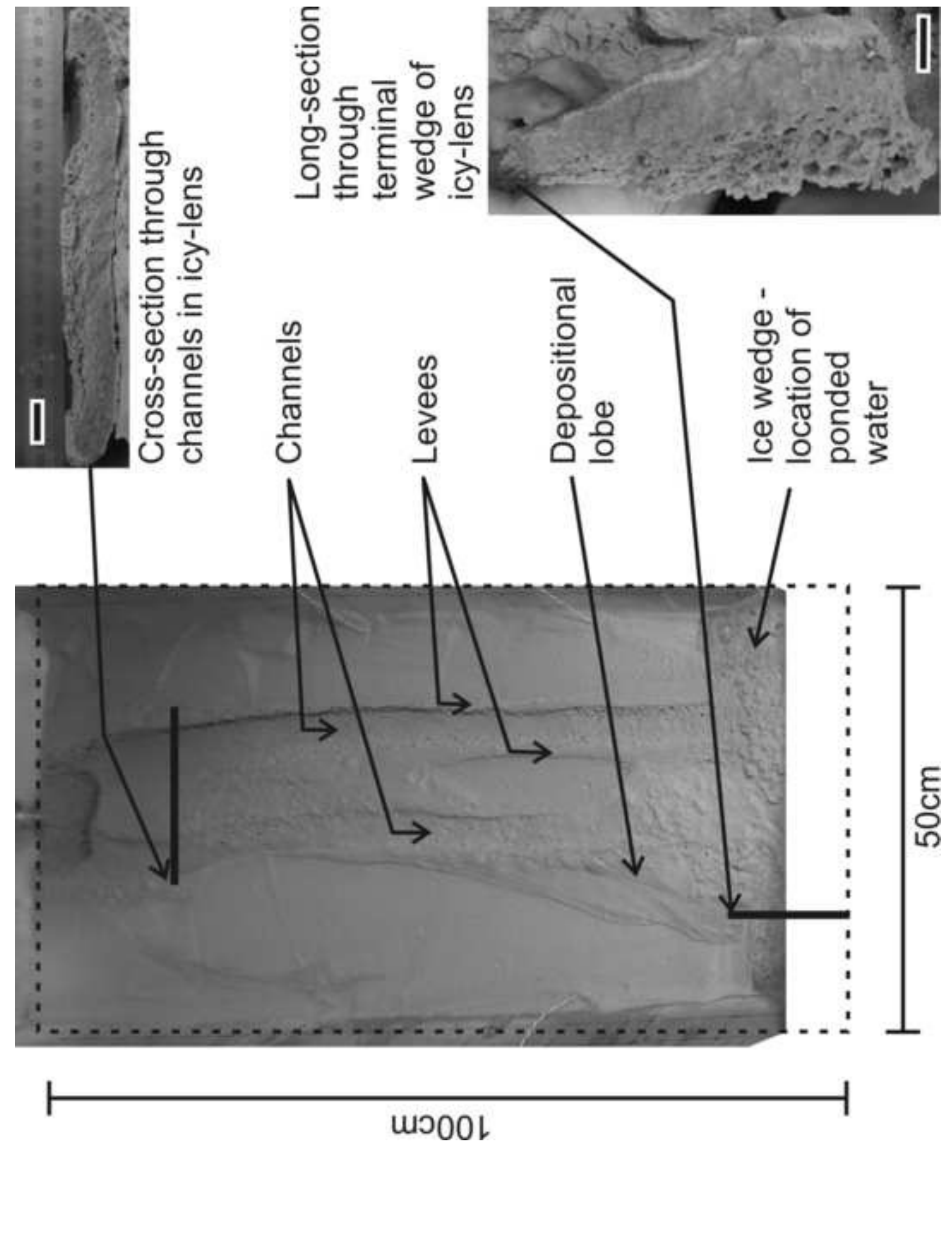




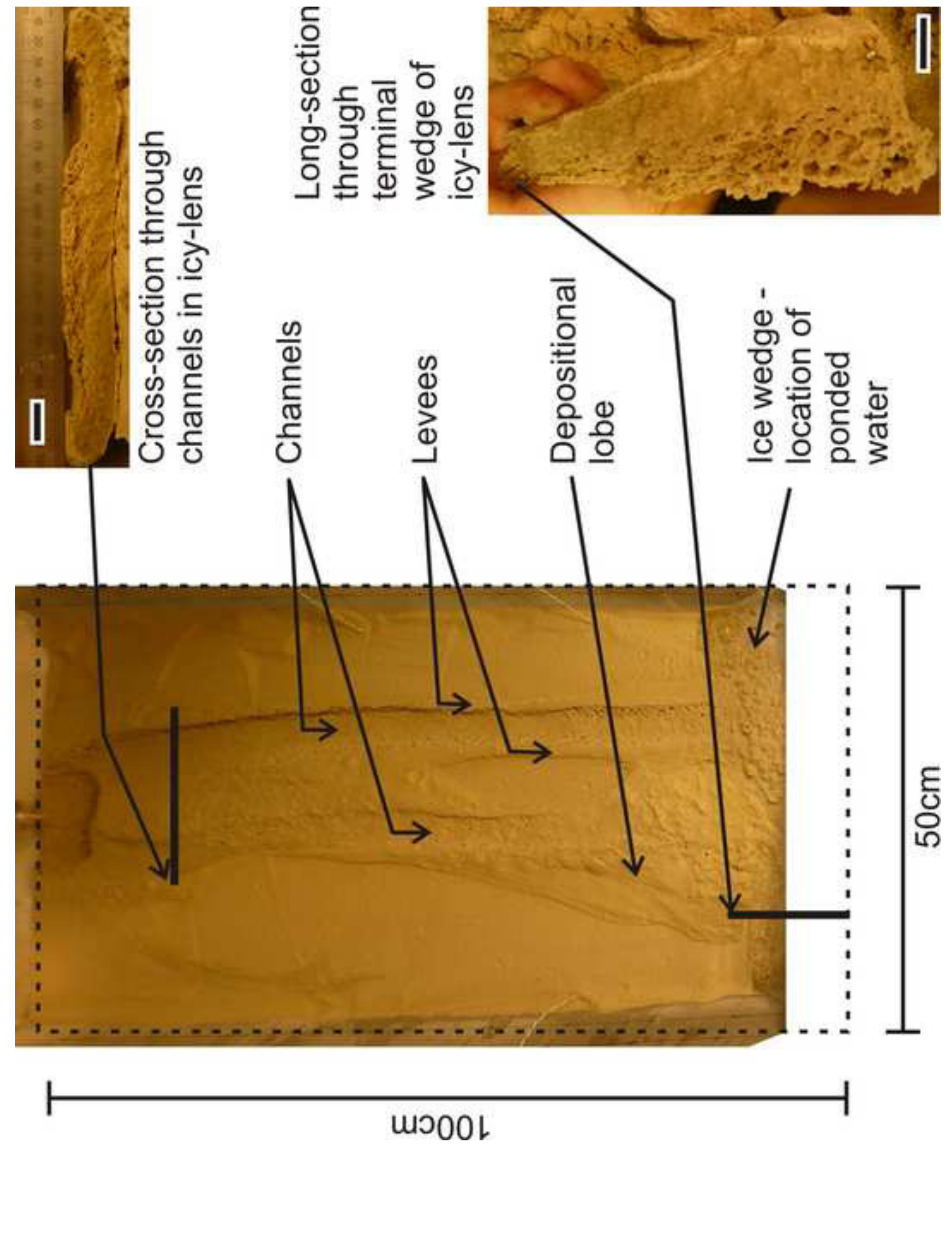




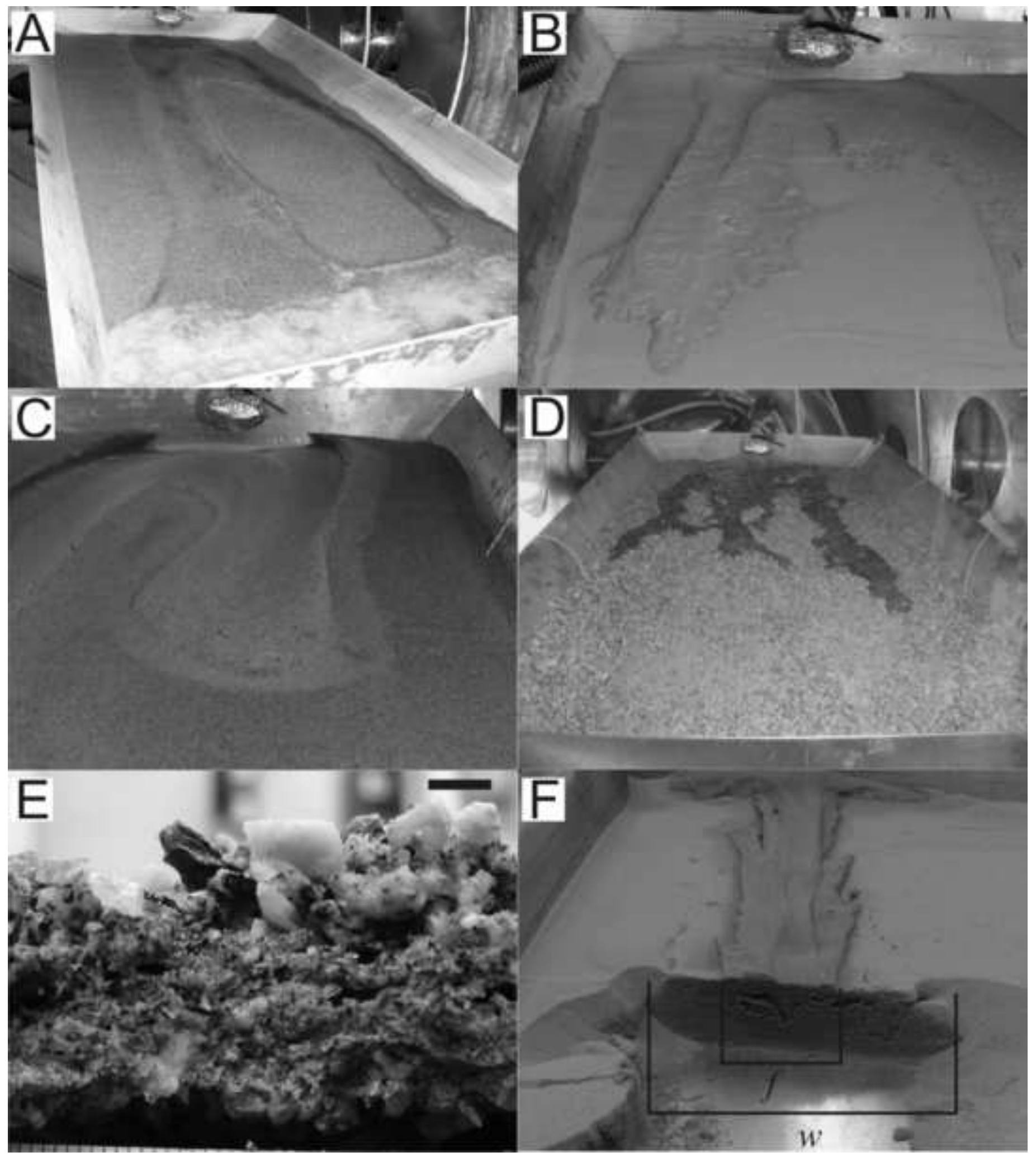




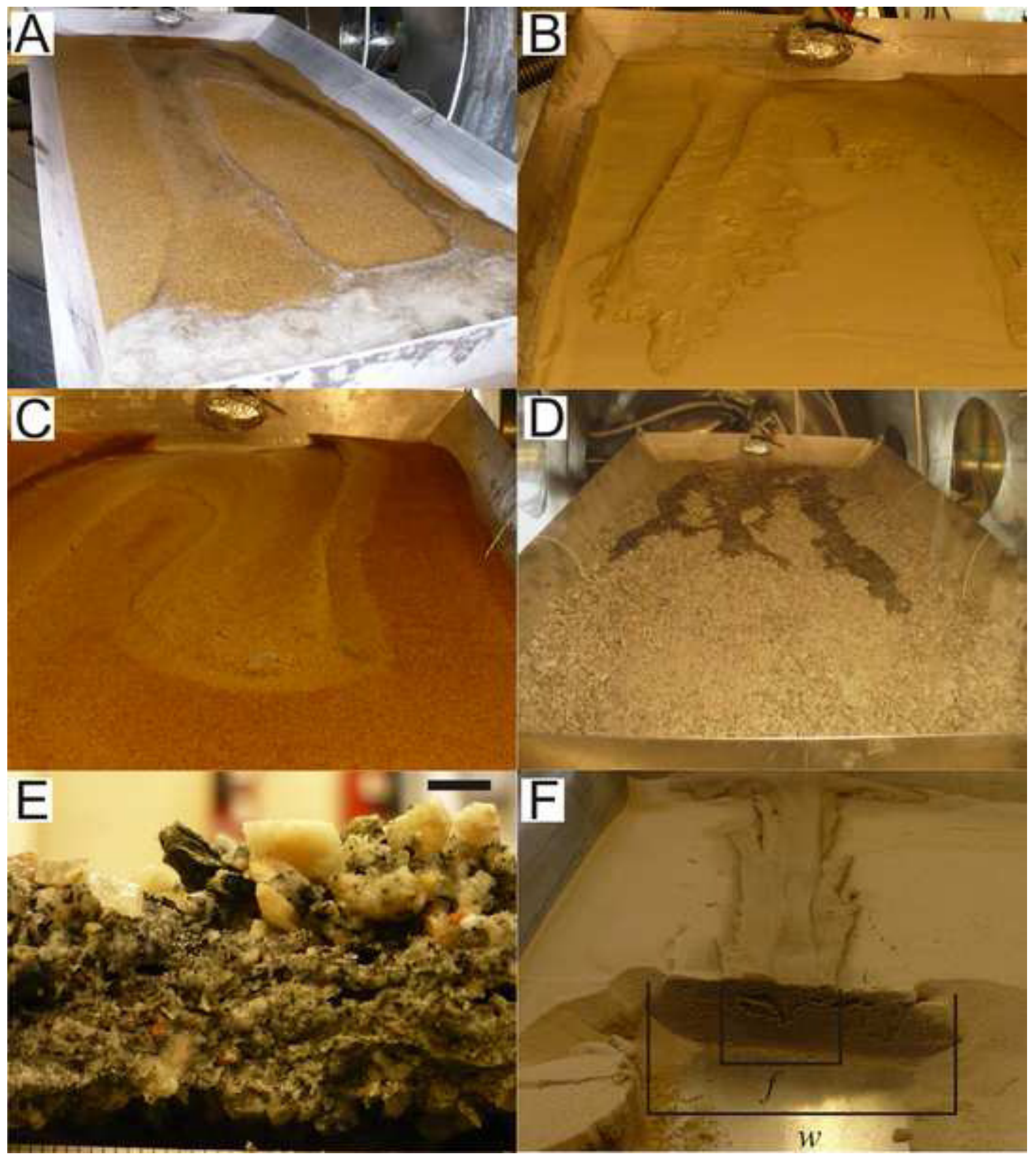




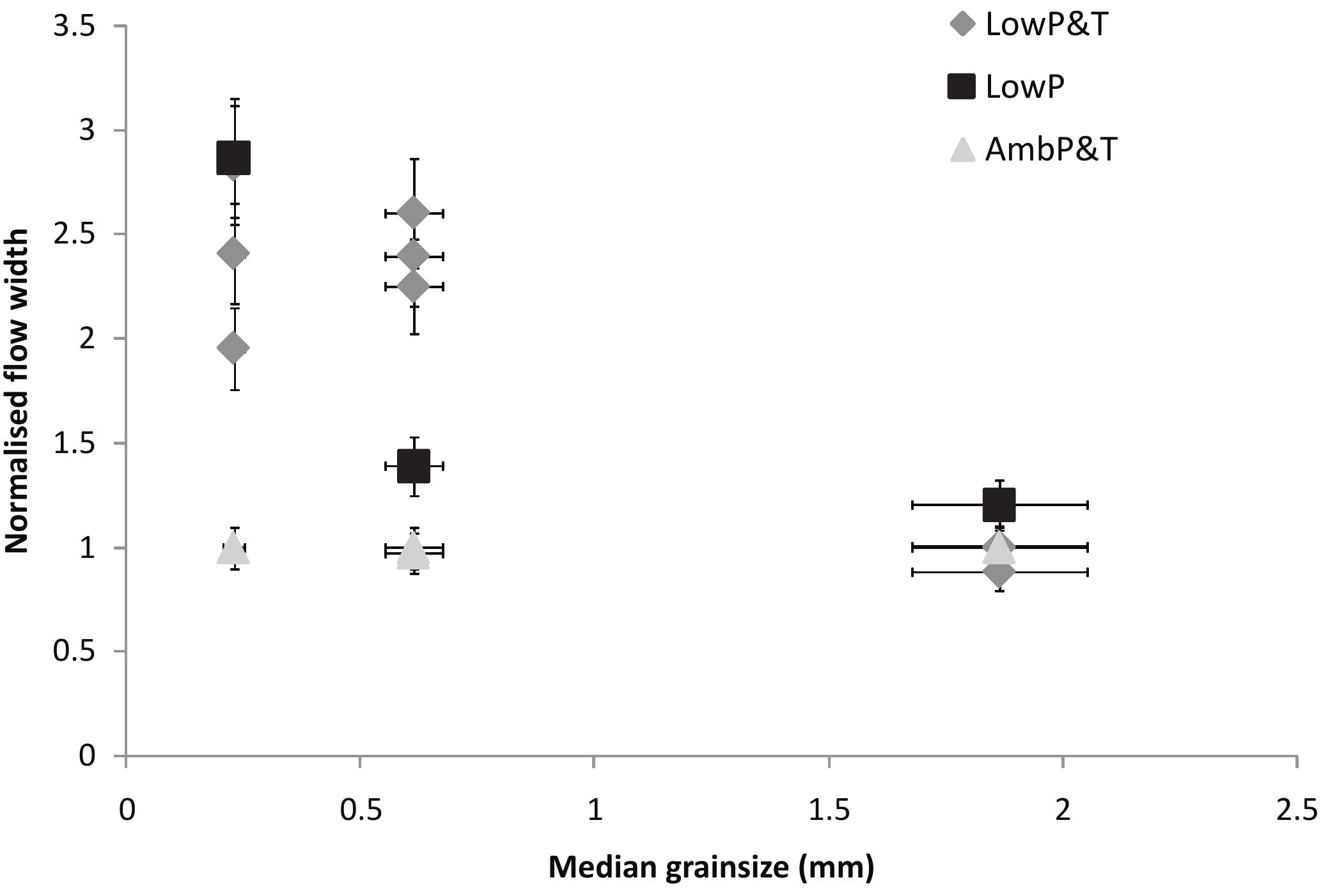




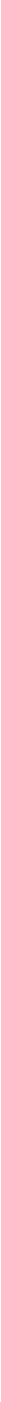




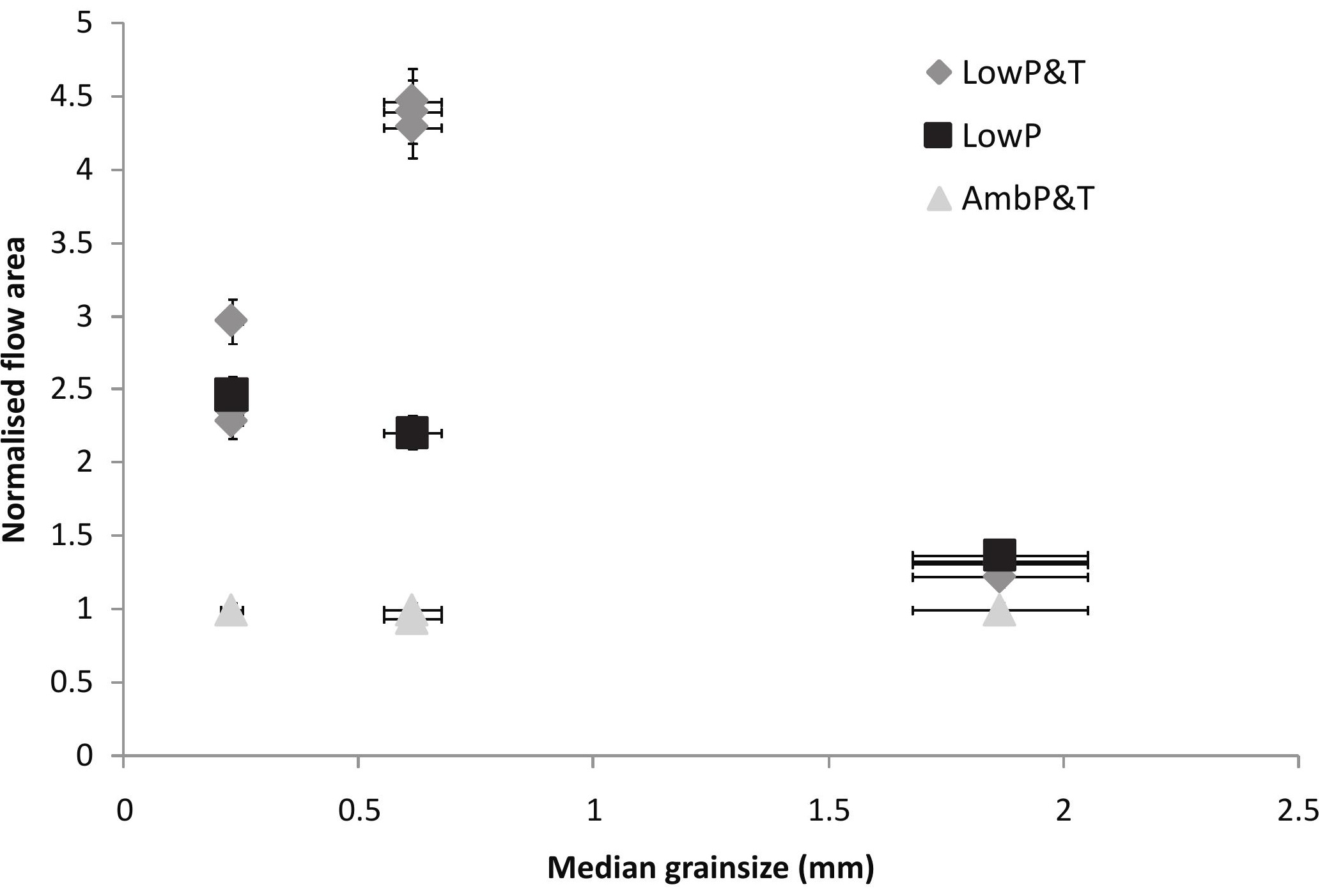




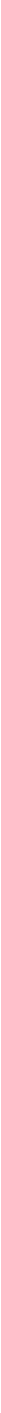


$<-15$

$-15--10$

$-10--5$

$-5-0$

$0-5$

$5-10$

$10-15$

$>15$

Ice thickness

Deposition volume

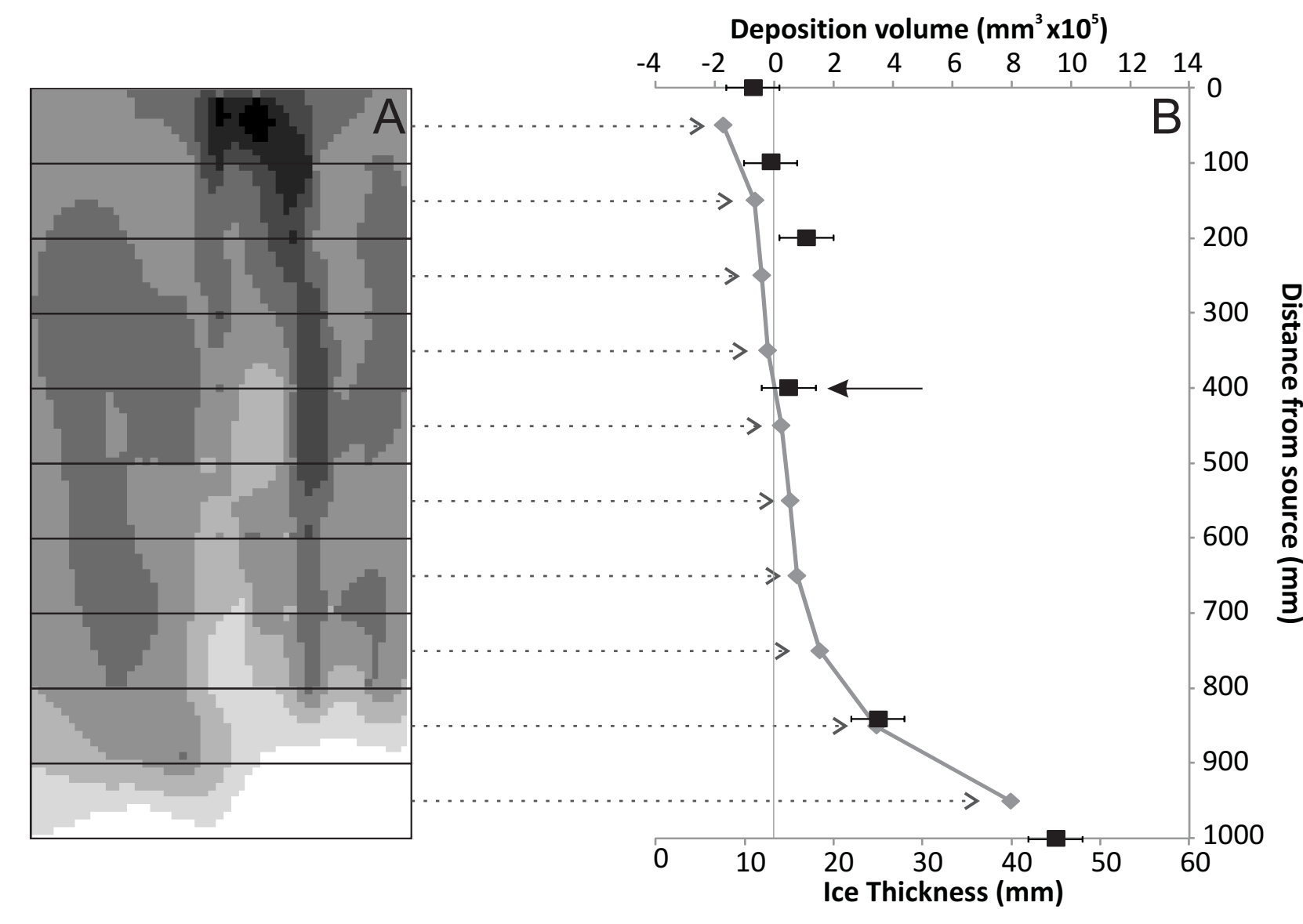




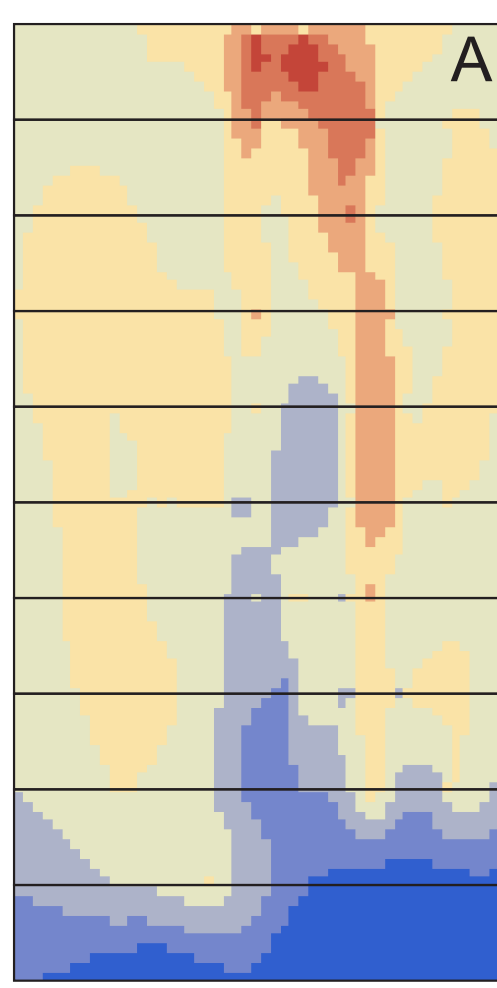

Deposition volume $\left(\mathrm{mm}^{3} \times 10^{5}\right)$

$\begin{array}{llllllllll}-4 & -2 & 0 & 2 & 4 & 6 & 8 & 10 & 12 & 14\end{array}$

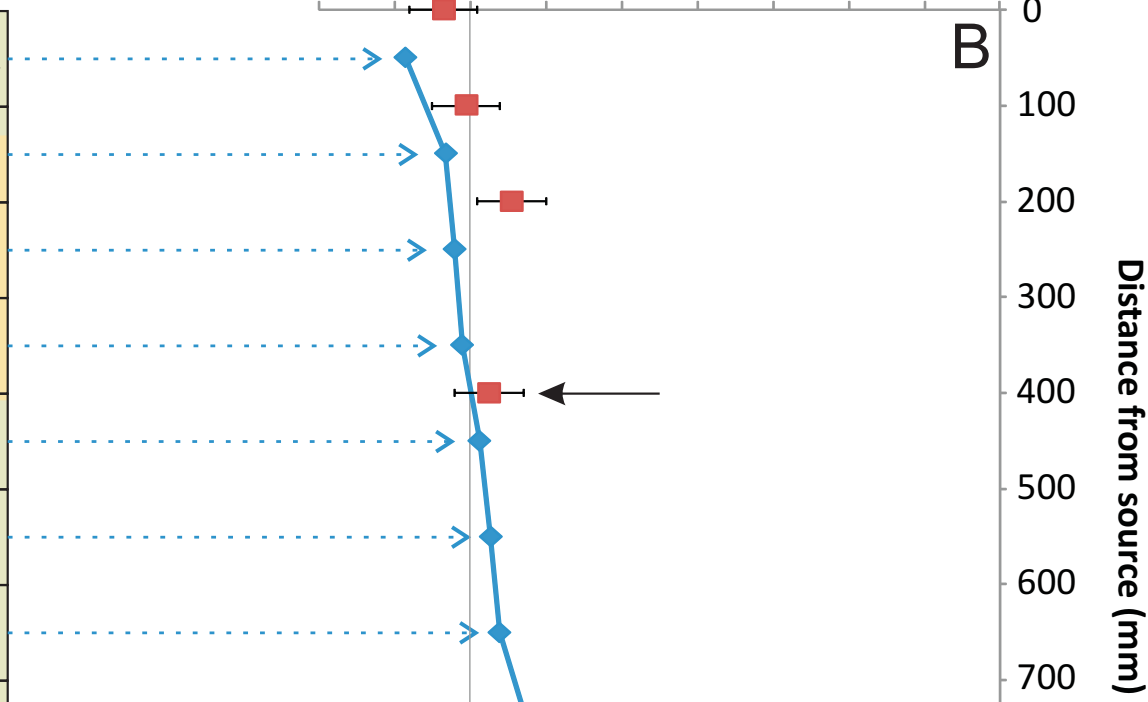


Figure 9

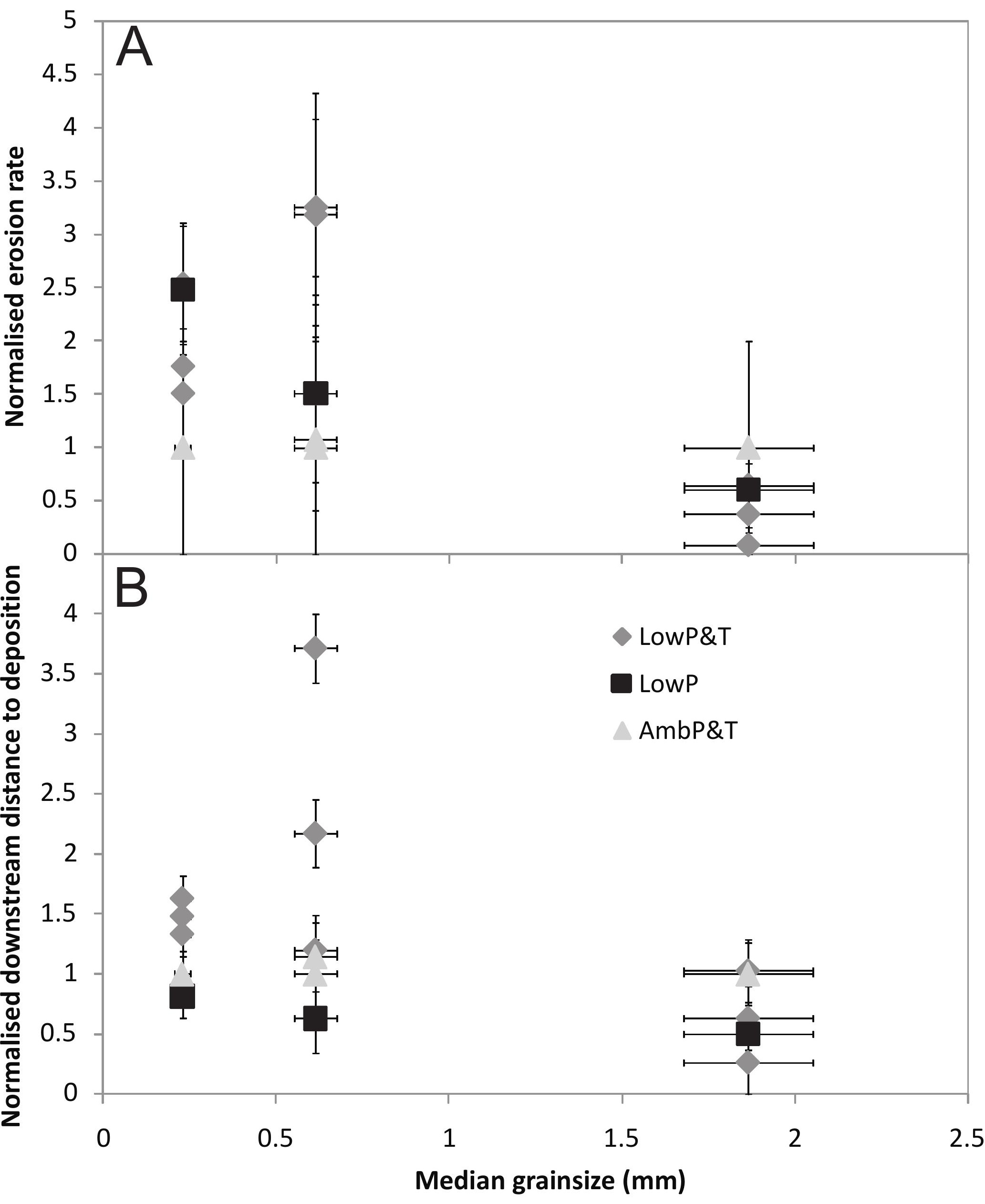


Figure 9 - colour

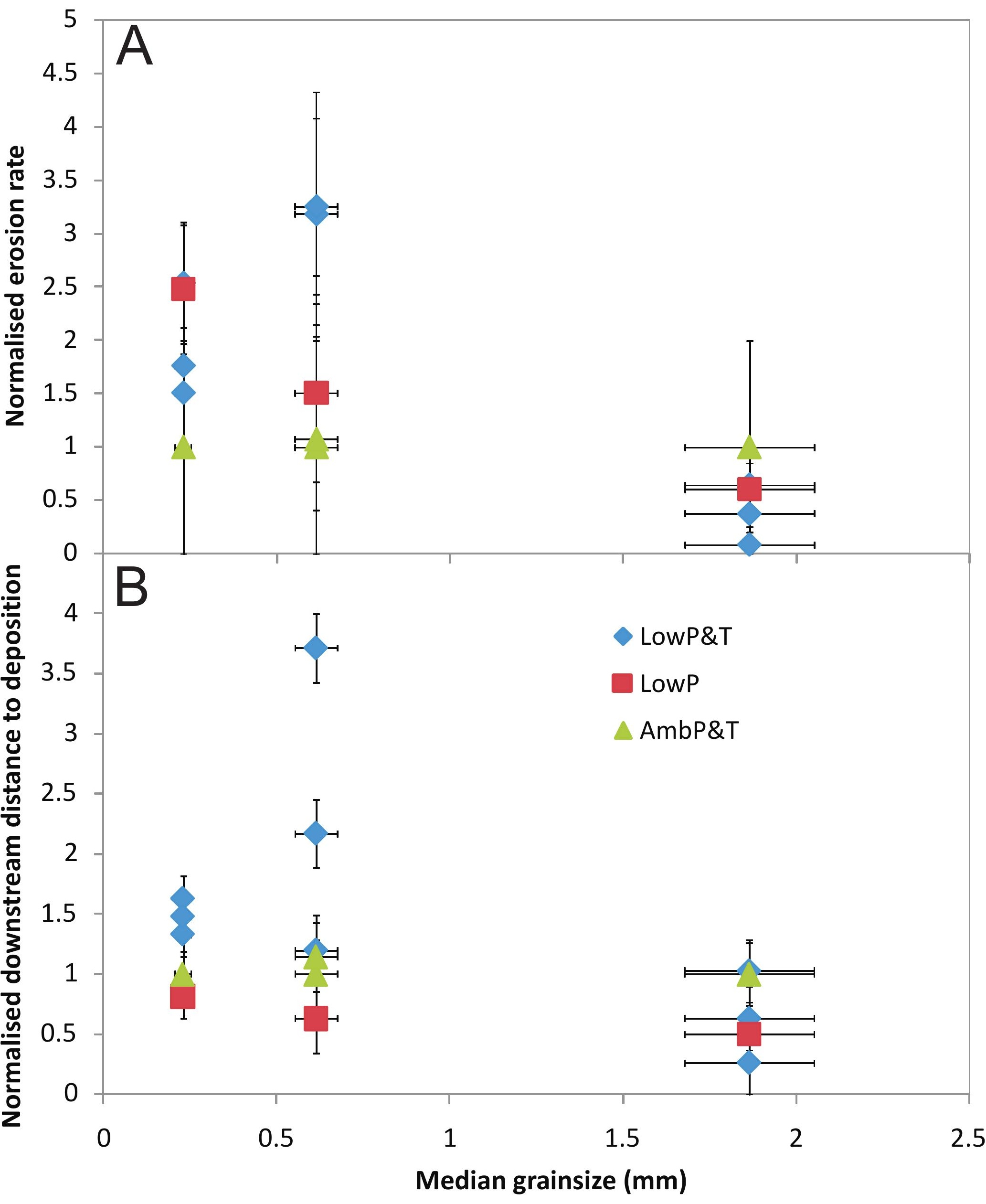




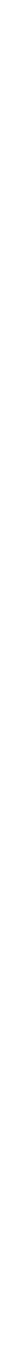

Median grainsize $(\mathrm{mm})$ 


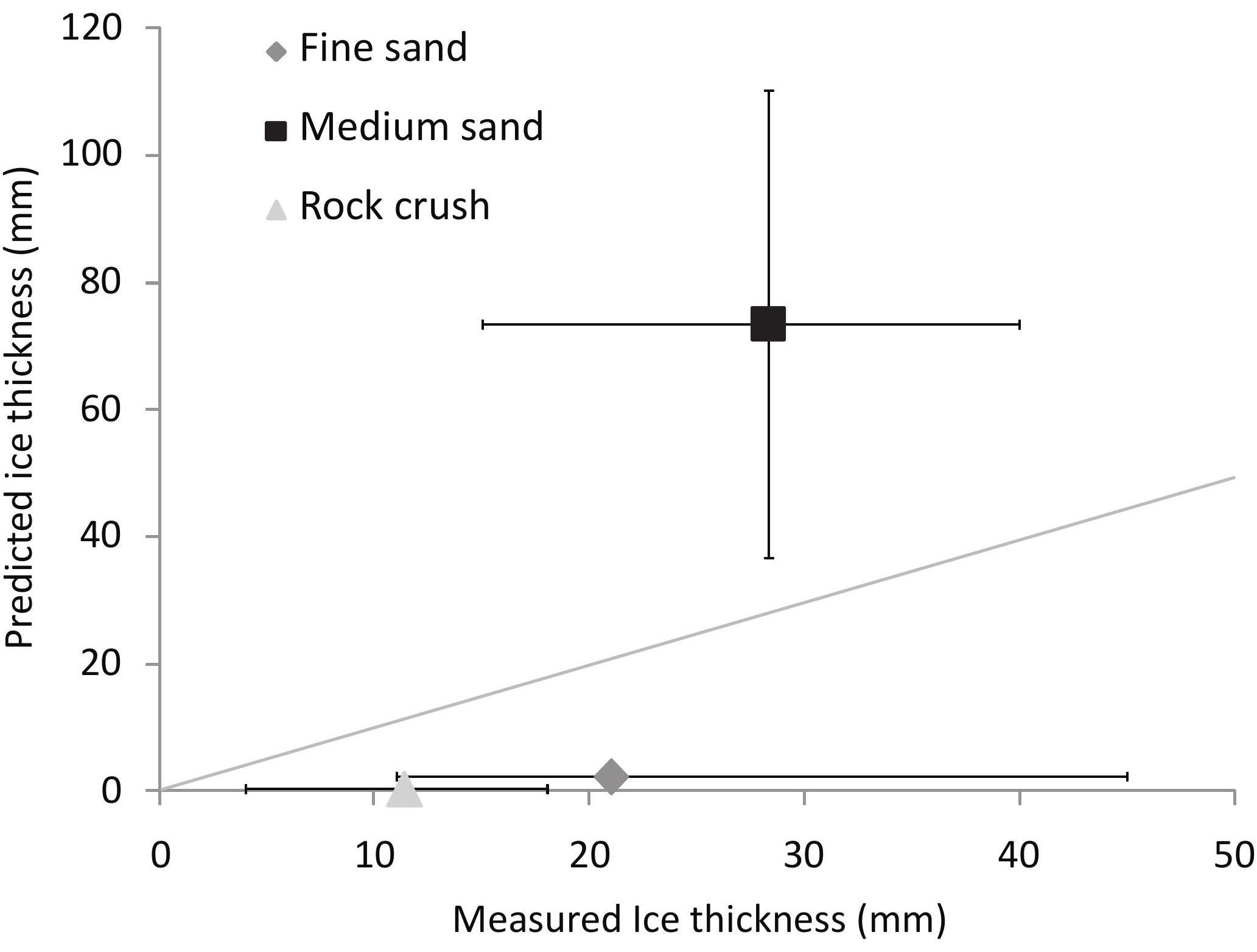




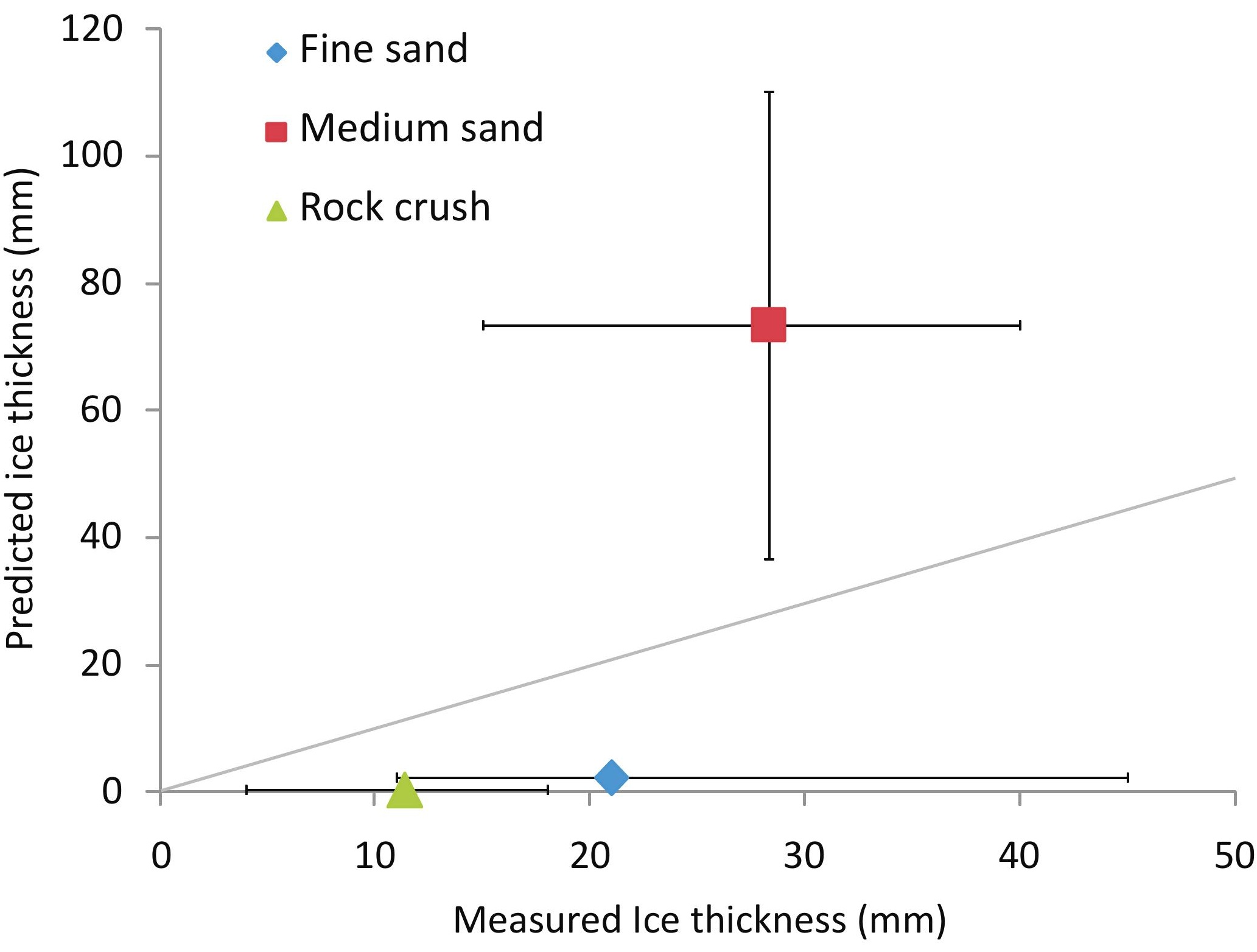


Figure 12

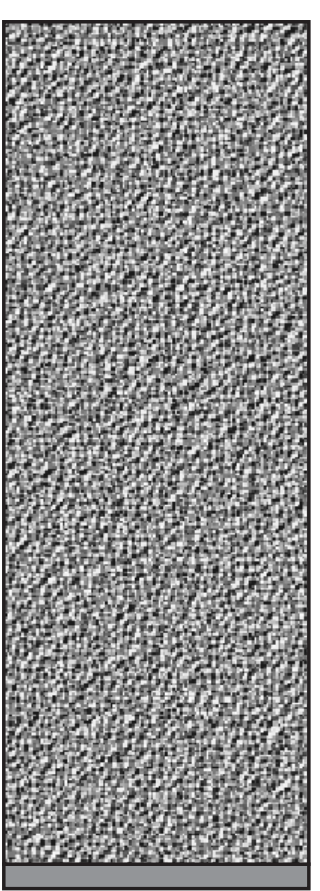

$t_{0}$

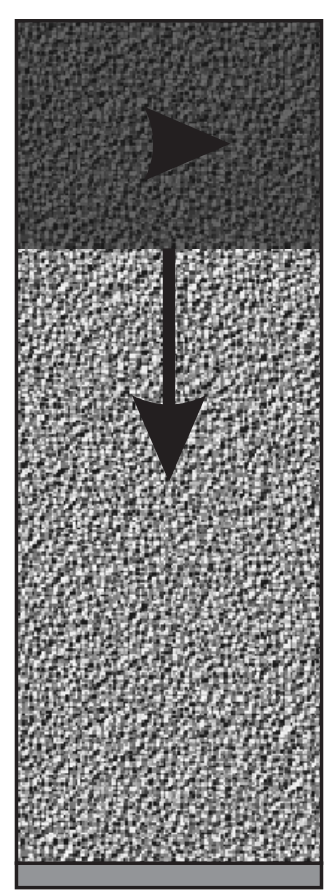

$t_{1}$

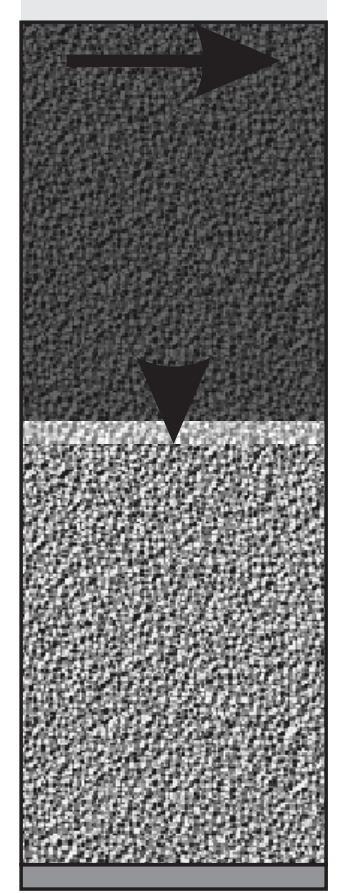

$t_{2}$

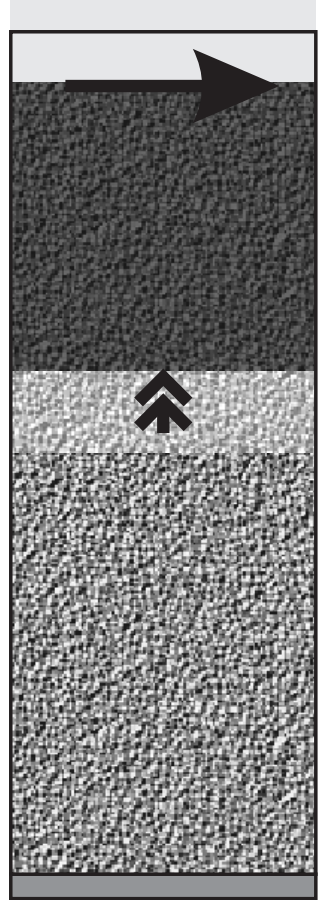

$t_{3}$

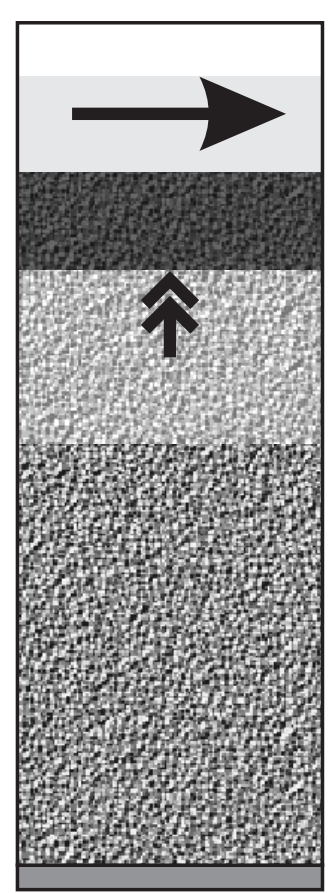

$t_{4}$

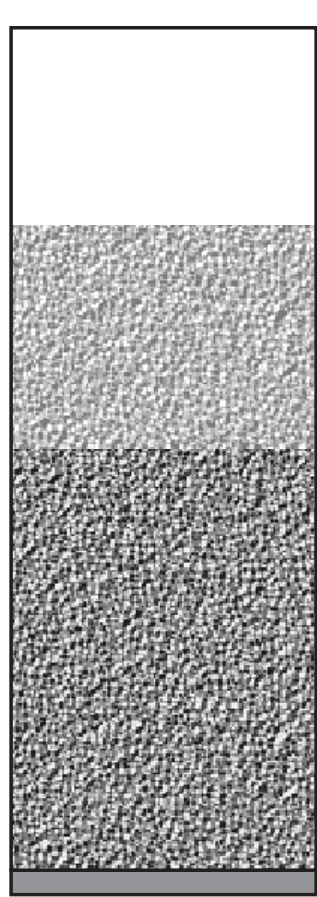

$t_{5}$ 


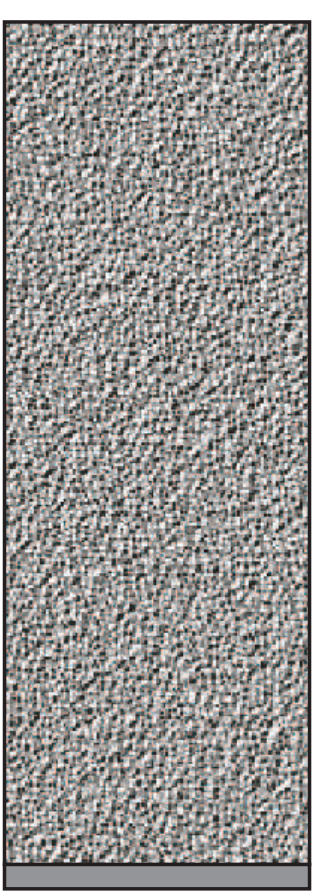

$t_{0}$

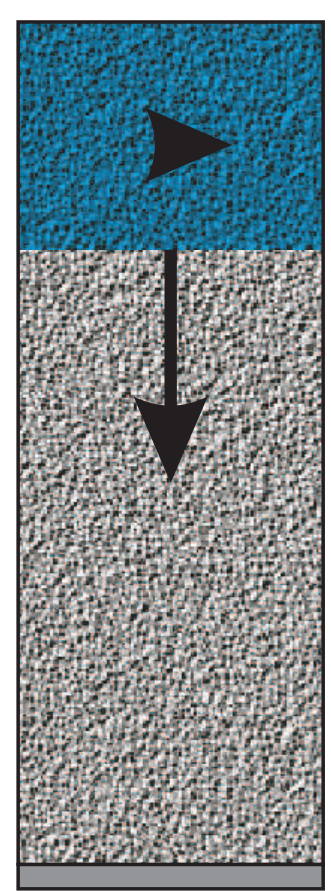

$t_{1}$

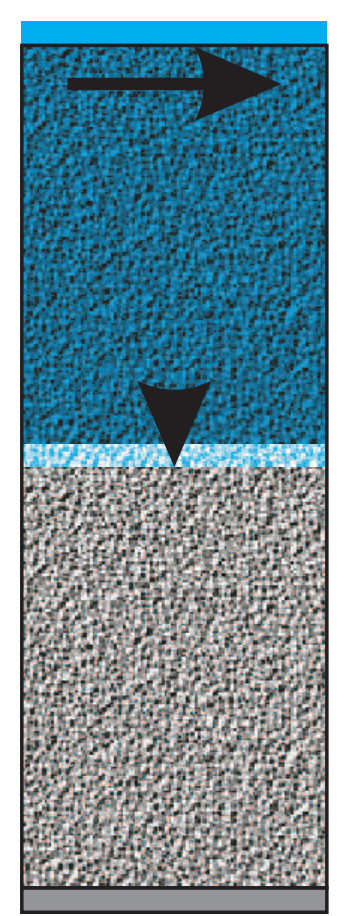

$t_{2}$

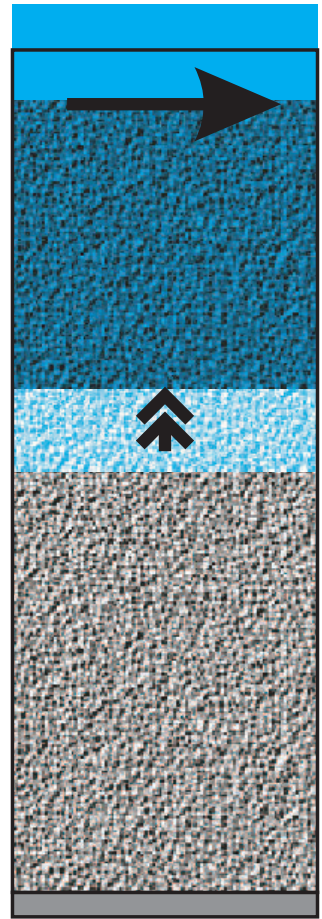

$t_{3}$

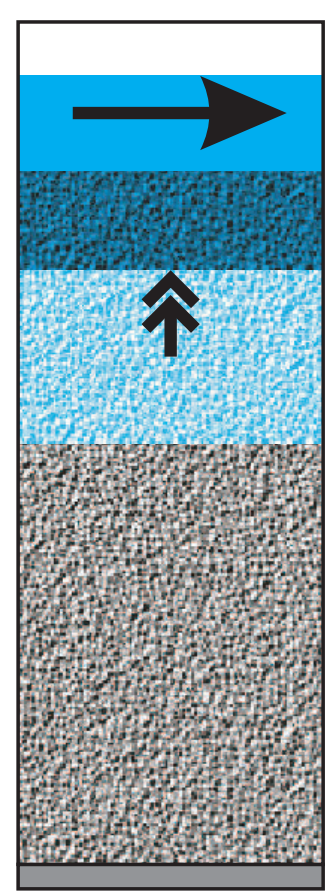

$t_{4}$

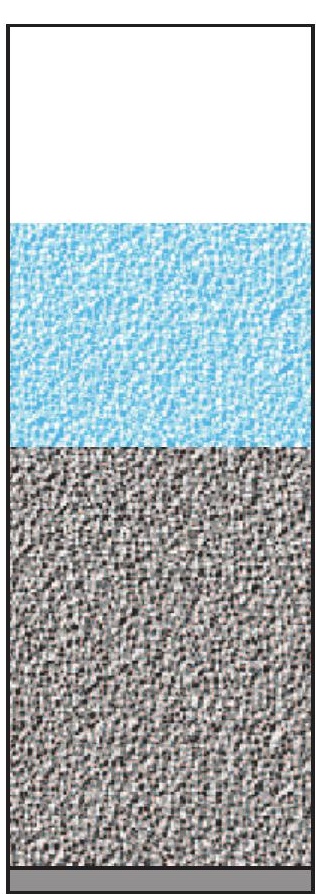

$t_{5}$ 


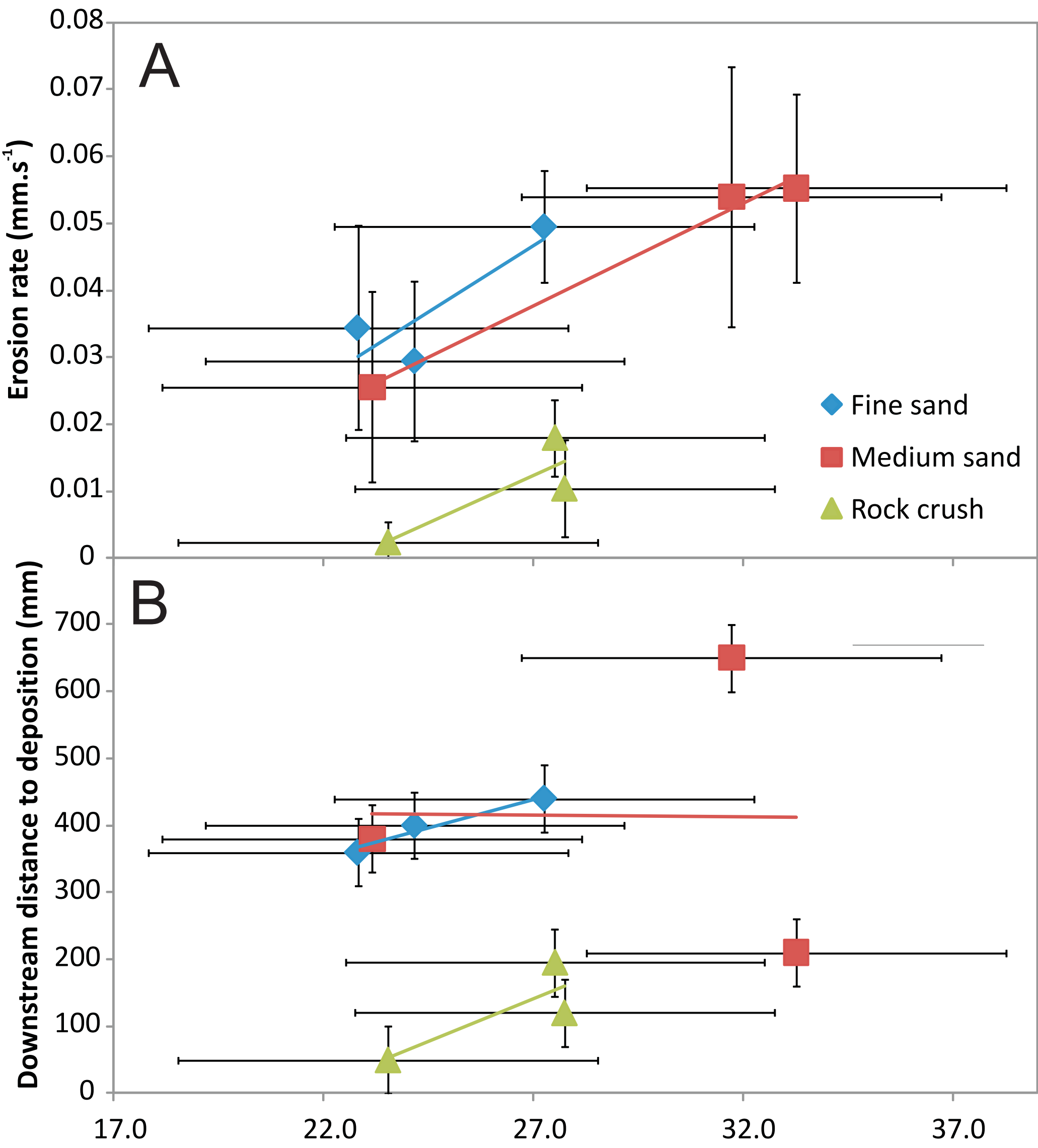

Difference in temperature between water and sediment $\left({ }^{\circ} \mathrm{C}\right)$ 


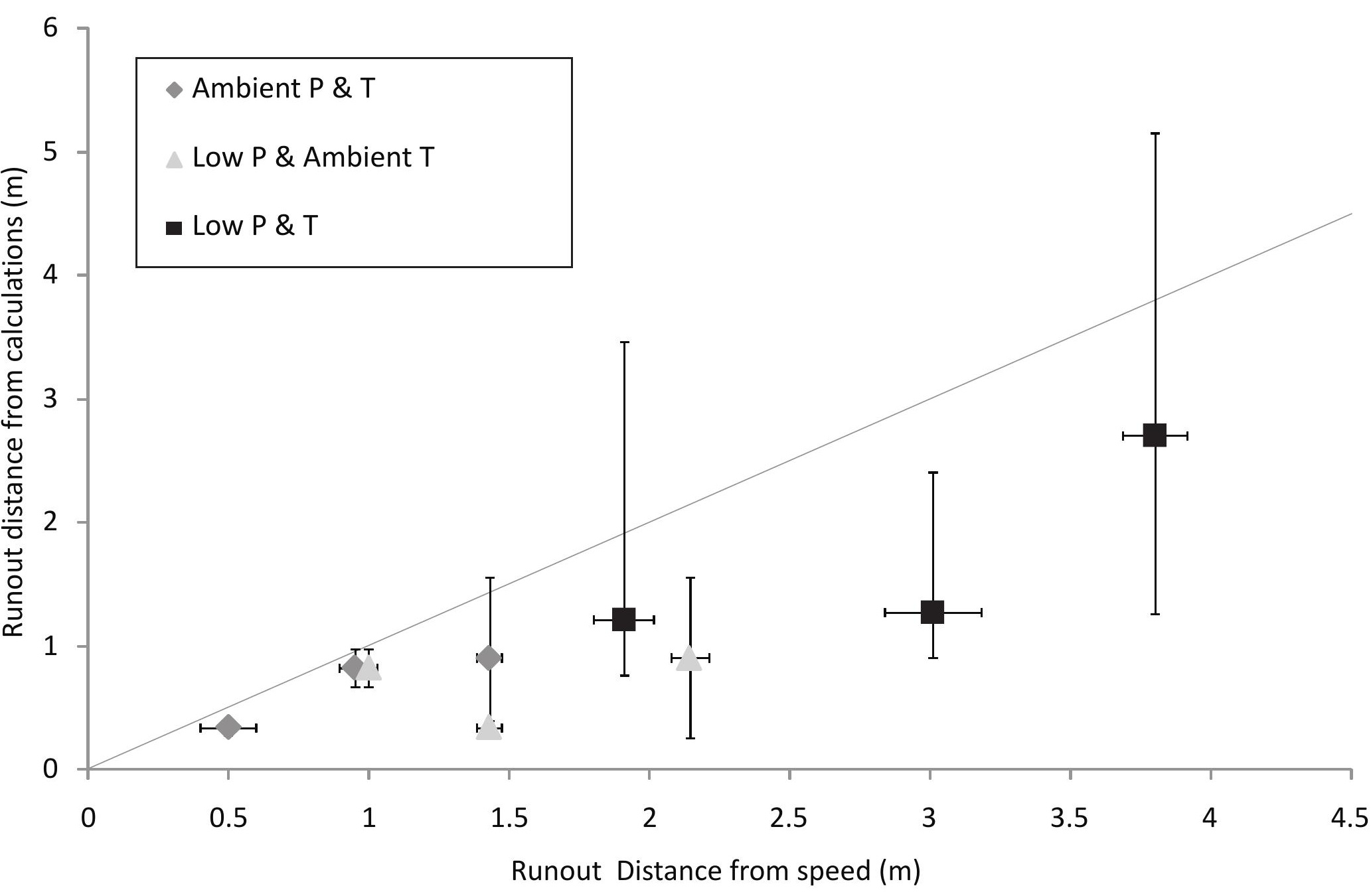




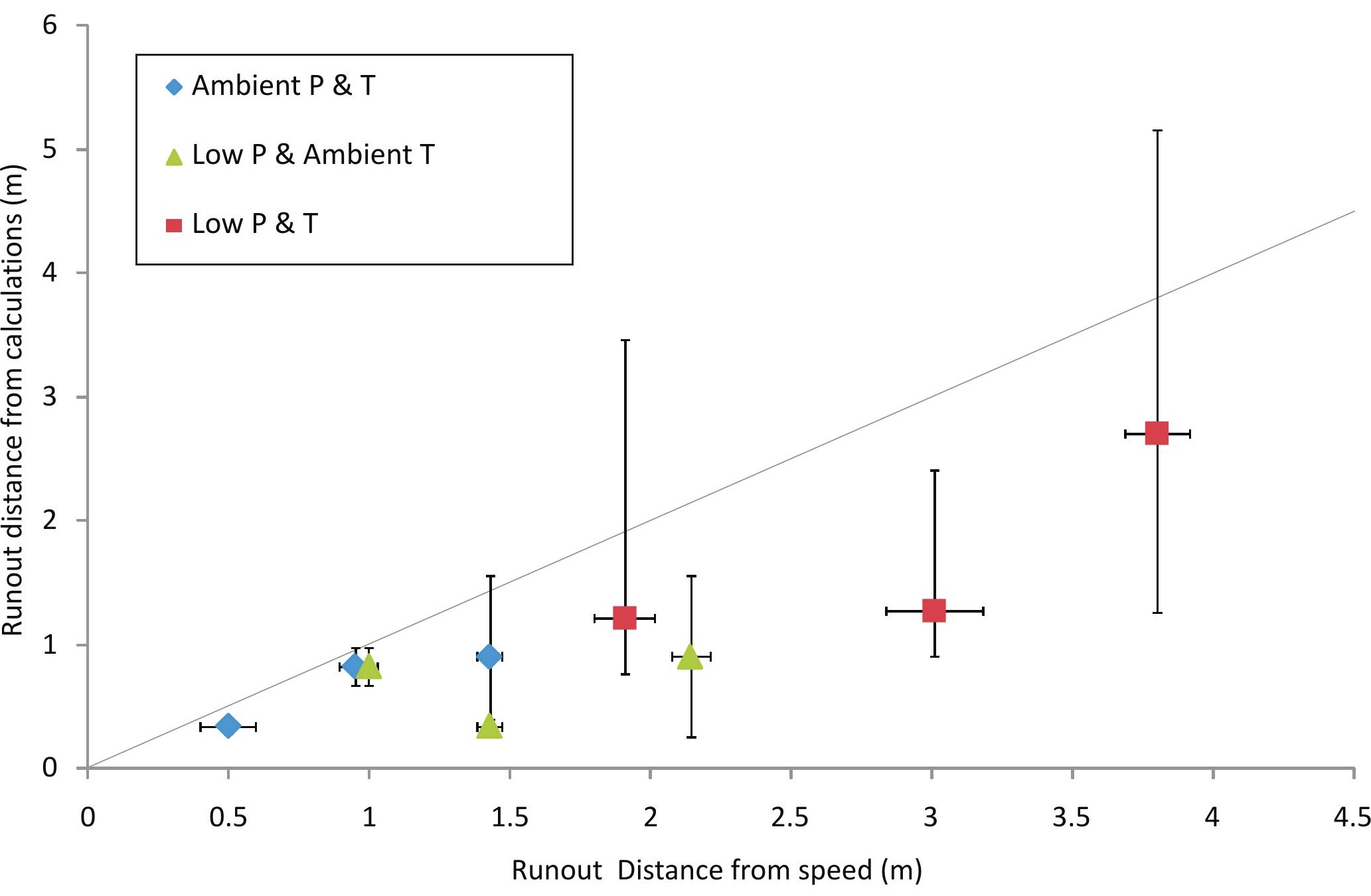


Table 1: Sediment characterisation. ${ }^{\mathrm{a}}$

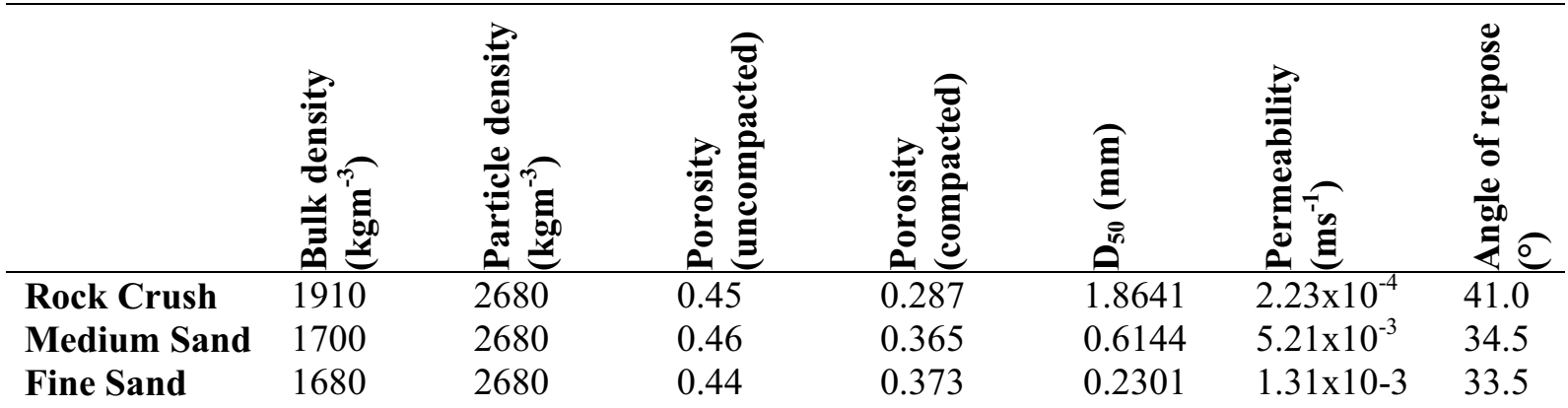

${ }^{a}$ Where $\mathrm{D}_{50}$ represents the modal grain size of the distribution 


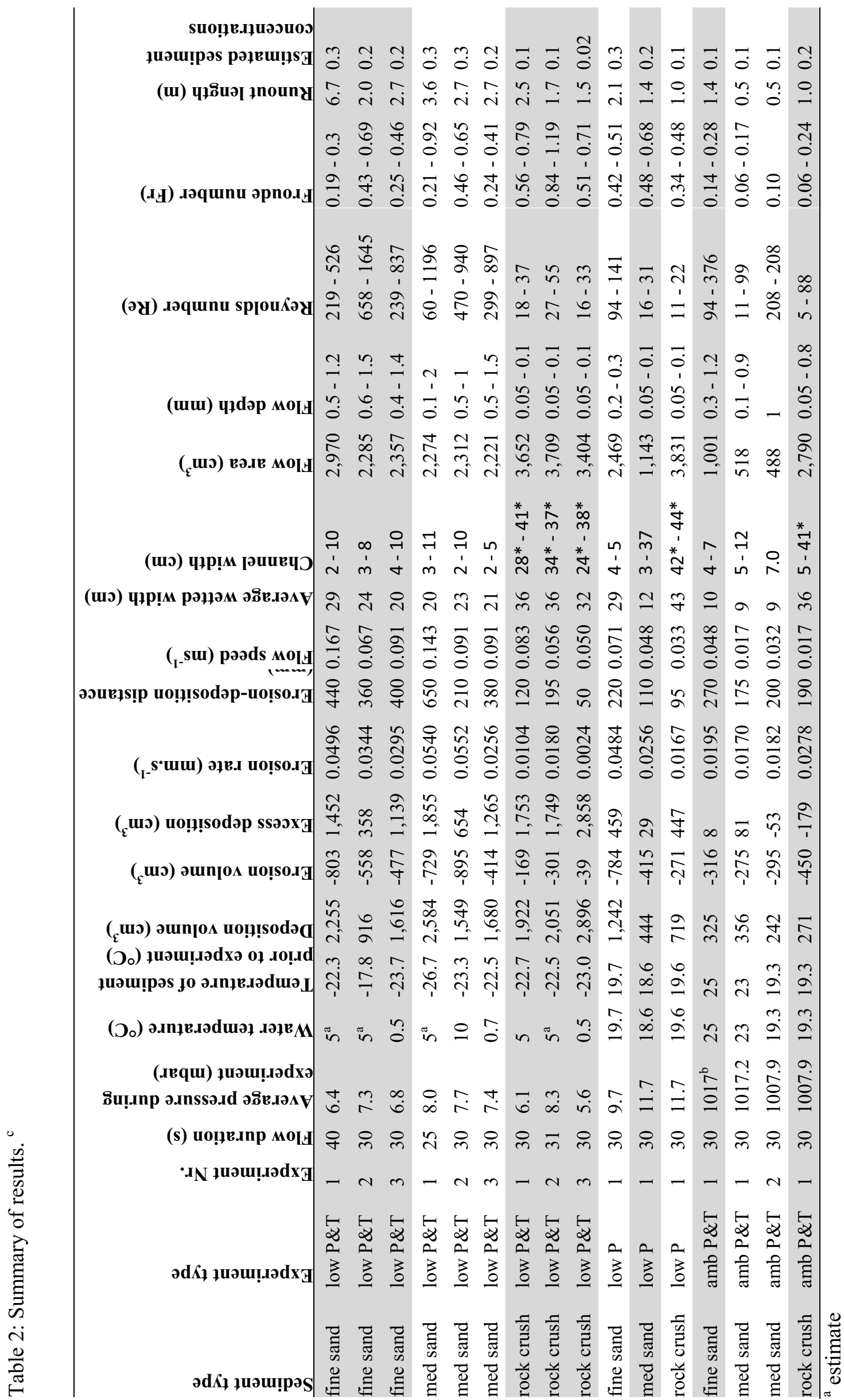




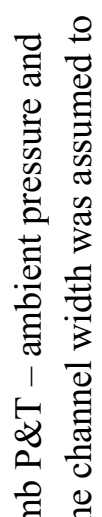

童

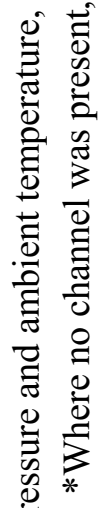

芯

官 豆

a is 0

zo

을

!

讷

宅

客言䒕

空 学

용

田.

ㄴ.

石 $\simeq$

1象总

$\approx$

合 छ

응

ㅁ. है

च

도를

馬

馬

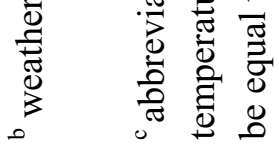


Table 3: Calculation of depth to freezing front and other flow parameters. ${ }^{a}$

\begin{tabular}{|c|c|c|c|}
\hline & $\begin{array}{l}\text { Medium } \\
\text { Sand }\end{array}$ & $\begin{array}{l}\text { Fine } \\
\text { Sand }\end{array}$ & $\begin{array}{l}\text { Rock } \\
\text { Crush }\end{array}$ \\
\hline Pore space (mm) & 0.61 & 0.23 & $0.23 *$ \\
\hline Energy to lose to reduce from $5 \mathrm{C}$ to zero $\left(\mathrm{J} / \mathrm{m}^{2}\right)$ & 12841 & 4842 & 4842 \\
\hline Enthalpy of fusion $\left(\mathrm{J} / \mathrm{m}^{2}\right)$ & 203400 & 76900 & 77200 \\
\hline Total Energy to lose $\left(\mathrm{J} / \mathrm{m}^{2}\right)$ & 216306 & 81788 & 82018 \\
\hline Average temperature difference $(K)$ from Table 2 & 15.2 & 17.2 & 17.7 \\
\hline Rate of energy loss from Fourier's Law $\left(\mathrm{J} / \mathrm{s} \cdot \mathrm{m}^{2}\right)$ & 14452 & 43374 & 44635 \\
\hline Time to freeze (s) & 15.0 & 1.9 & 1.8 \\
\hline Depth to freezing front $(\mathrm{mm})$ & 78 & 2.5 & 0.4 \\
\hline Depth to freezing front as percentage of D50 & $1.3 \times 10^{4}$ & $1.0 \times 10^{3}$ & 100 \\
\hline
\end{tabular}

${ }^{\text {a }}$ The pore space was estimated to be equivalent to the modal grain size for each material $\left(D_{50}\right) *$ Pore space of rock crush assumed to be much smaller than its $\mathrm{D}_{50}$, as a conservative estimate the pore space of the fine sand was used. 


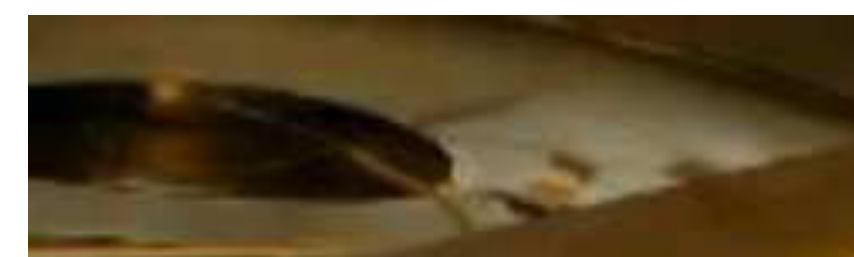

\section{8.}

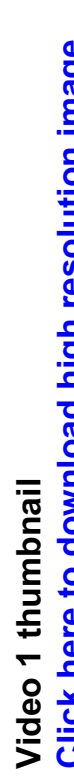


Click here to download Supplementary Material for on-line publication only: v1_090715_001_and_090619_001_a.wmv 


\section{Video 2}

Click here to download Supplementary Material for on-line publication only: v2_lowPT_med_090519_001.mp4 


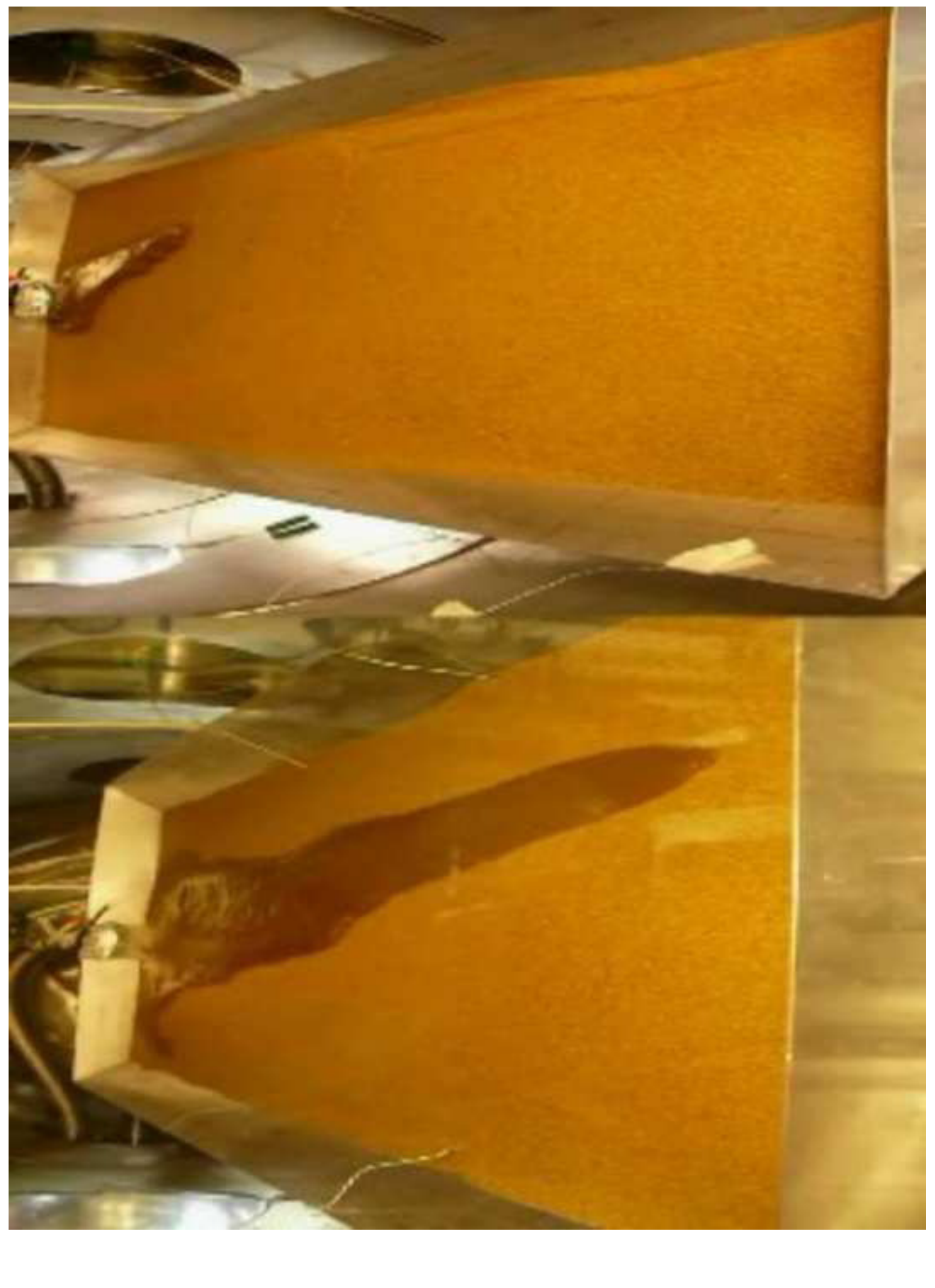


Click here to download Supplementary Material for on-line publication only: v3_090710_001_and_090902_002_a.wmv 


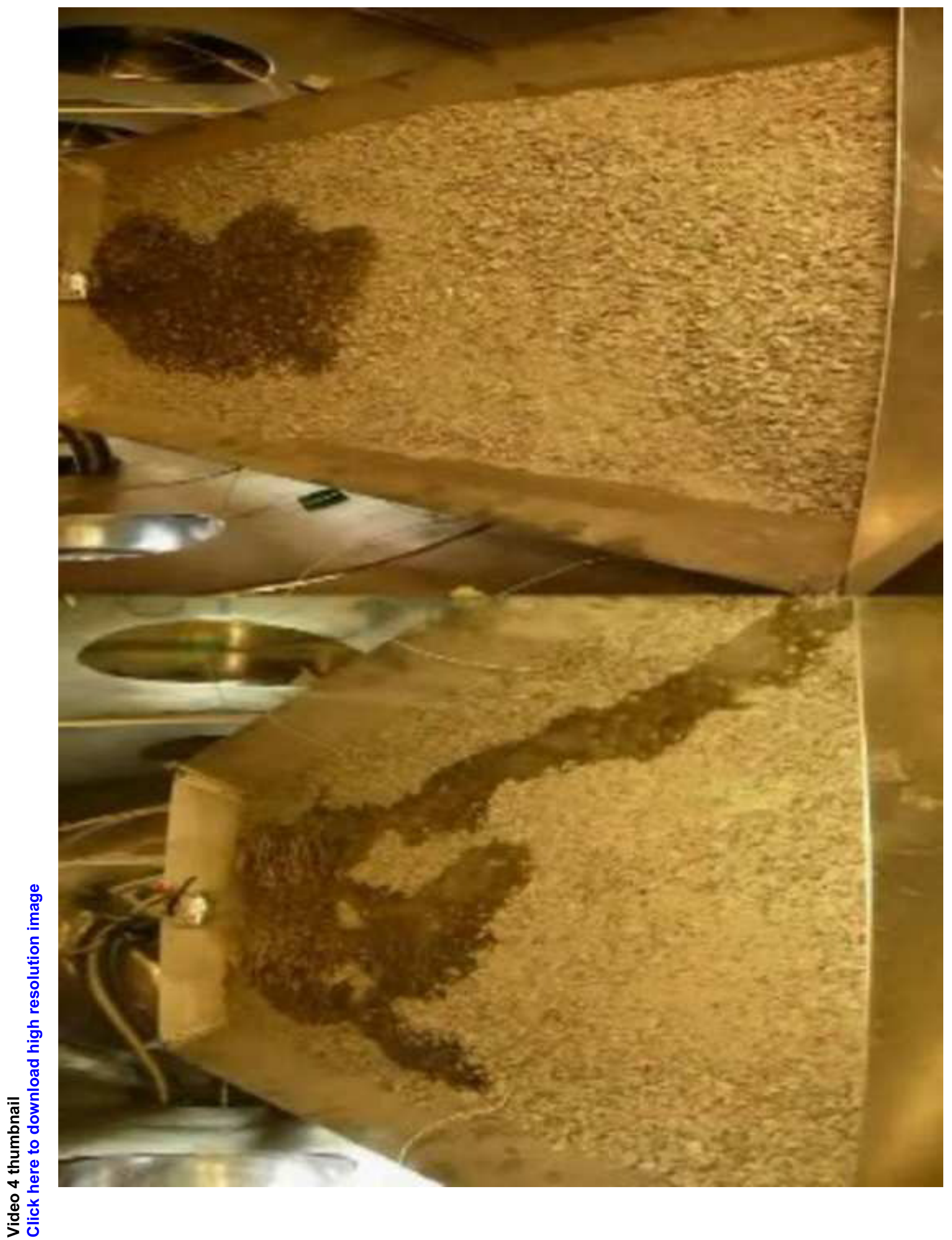




\section{Video 4}

Click here to download Supplementary Material for on-line publication only: v4_090902_001_and_090612_001_a.wmv 


\section{Video 5}

Click here to download Supplementary Material for on-line publication only: v5_lowPT_rc_090714_001.mp4 
Click here to download Supplementary Material for on-line publication only: v6_090514_001_Ihs_figure.wmv 